\title{
Mid-infrared Nanoantennas as Ultrasensitive Vibrational Probes Assisted by Machine Learning and Hyperspectral Imaging
}

\section{Zhihao Ren}

National University of Singapore https://orcid.org/0000-0002-2520-0784

\section{Zixuan Zhang}

National University of Singapore

Jingxuan Wei

National University of Singapore https://orcid.org/0000-0003-0295-3764

\section{Bowei Dong}

National University of Singapore

Chengkuo Lee ( $\nabla$ elelc@nus.edu.sg)

National University of Singapore

\section{Methods Article}

Keywords: Infrared Spectroscopy, Biomolecular Screening, Microfluidic Channel, Molecular Recognition

Posted Date: March 1st, 2021

DOI: https://doi.org/10.21203/rs.3.rs-209363/v1

License: (c) (1) This work is licensed under a Creative Commons Attribution 4.0 International License. Read Full License 


\section{Mid-infrared Nanoantennas as Ultrasensitive Vibrational Probes}

2 Assisted by Machine Learning and Hyperspectral Imaging

4 Zhihao Ren ${ }^{1,2,3,5}$, Zixuan Zhang ${ }^{1,2,3,5}$, Jingxuan Wei ${ }^{1,2,3,5}$, Bowei Dong ${ }^{1,2,3}$, Chengkuo Lee ${ }^{1,2,3,4^{*}}$

5

$6{ }^{1}$ Department of Electrical and Computer Engineering, National University of Singapore, 4

7 Engineering Drive 3, Singapore 117576, Singapore.

$8{ }^{2}$ Center for Intelligent Sensors and MEMS (CISM), National University of Singapore, 4

$9 \quad$ Engineering Drive 3, Singapore 117576, Singapore.

$10 \quad{ }^{3}$ NUS Suzhou Research Institute (NUSRI), Suzhou 215123, China.

$11{ }^{4}$ NUS Graduate School - Integrative Sciences and Engineering Programme (ISEP), National

12 University of Singapore, 21 Lower Kent Ridge Road, Singapore 119077, Singapore.

$13{ }^{5}$ These authors contributed equally: Zhihao Ren, Zixuan Zhang, Jingxuan Wei

14 *E-mail: elelc@nus.edu.sg 


\section{ABSTRACT:}

25 Infrared (IR) Spectroscopy has been developed for centuries and has been widely used to identify 26 molecular structure from the massive information provided by IR fingerprint absorption, reflecting 27 the vibration energy of the chemical bond. Due to the intrinsically weak light-matter interaction, 28 IR spectroscopy serves low sensitivity and sizeable optical interaction length ( $\sim \mathrm{mm}$ to $\sim \mathrm{cm})$ 29 compared with other optical probes like Raman, florescent, and refractometry technology, which 30 hinder the applications for ultra-sensitive biomolecular screening. Here, we reported a new type 31 of IR spectroscopy by wavelength gradient hook nanoantenna integrated with microfluidic channel, 32 enhancing the IR molecular absorption and bringing in refractometry function with ultrathin $(\sim 100$ $33 \mathrm{~nm}$ ) optical interaction length. With the proof-of-concept demonstration of molecular recognition 34 of mixed alcoholic liquids by machine learning and molecular fingerprint retrieving by 35 hyperspectral images in one-time data acquisition, our work paves the way to advance, small36 volume, real-time, ultra-sensitive, in-vitro biomolecular dynamic analysis in aqueous environment. 


\section{Introduction}

47 Molecular identification of gas $^{1}$, liquid $^{2}$, and biomolecules ${ }^{3}$ is a fundamental requirement for various applications such as environmental monitoring, healthcare, clinical diagnosis, and

49 biological screening. The ideal methods to identify molecules are in-situ detection of the chemical

50 structures for molecules in a label-free and non-destructive manner with fast response time and

51 high sensitivity. Optical approaches are suitable methods for molecule screening in life science

52 thanks to remote, real-time, and sensitive detection with the aid of the development of microscopy

53 technology. Mid-infrared (MIR) fingerprint absorption, reflecting the genetic information of

54 molecule structures in chemical bonds and functional groups, provides natural optical probes for

55 molecule identification. Harnessing fingerprint absorption, IR spectroscopy offers a solution for

56 non-invasive, non-destructive, label-free, and real-time recognition and monitoring of molecules,

57 especially in the mixture. However, traditional IR spectroscopy technology is limited by the large

58 optical path $(\sim \mathrm{mm}$ to $\sim \mathrm{cm})$ due to weak light-matter interaction in MIR, hindering the sensing

59 performance compared with other optical probes technologies such as $\operatorname{Raman}^{4}$, fluorescent ${ }^{5}$, and

60 refractometry ${ }^{6}$

61 To solve this problem, A. Hartstein et al. observed the enhancement of the IR absorption from

62 molecular monolayer with thin metal overlayers in 1980, bringing the concept of surface-enhanced

63 infrared absorption spectroscopy (SEIRAS). ${ }^{7}$ Furthermore, with nanofabrication technology

64 development, artificially structured nanoantenna is proposed to enhance the IR fingerprint

65 absorption by tailored plasmonic resonance. ${ }^{8,9}$ This amplification effect caused by the strong

66 plasmon-phonon coupling between plasmonic nanoantennas (PNAs) resonance and molecular

67 vibration was well-explained by temporal coupled-mode theory (TCMT). ${ }^{10,11}$ Depending on

68 different coupling criteria, the different resonance line shape can be observed as electromagnetic 
69 induced transparent (EIT)-like, electromagnetic induced absorption (EIA)-like or Fano-like

70 resonance in the resonance spectrum. ${ }^{12,13}$ Thanks to the excellent field confinement at resonance

71 wavelength, PNA also serves as an ultra-sensitive refractometry sensor to capture the refractive

72 index of analytes by wavelength shifts (e.g., color change in visible light), which carries the

73 information of physical properties of molecules. ${ }^{14,15}$

74 Unfortunately, in the IR fingerprint region, the PNA signal of absorption changes and 75 wavelength shifts always comes together, hindering the usage of complementary information 76 about the physical and chemical properties of analytes for molecule identification. Machine 77 learning (ML) is a powerful tool for separating the data from different domains to achieve 78 enhanced pattern recognition. With the aid of machine learning, decoupling the IR fingerprint 79 absorption and refractive index change induced by molecules has been reported for monitoring of 80 protein dynamics ${ }^{16}$ and recognition of physiological bio-marker ${ }^{17}$. However, the molecular 81 recognition capabilities are still limited to two specific molecules due to the narrow bandwidth and 82 low sensitivity. To achieve global molecular identification, massive information is required for 83 machine-learning-based recognition from PNA sensors. Consequently, high sensitivity and 84 broadband detection range become two critical figure-of-merits (FOMs) that reflect the 85 performance of PNA sensors.

86 Since the plasmon-phonon coupling is induced by a localized electric field near the PNA 87 surface, the straightforward approach is to concentrate the molecule to the active area, which is the 88 hot spot of the electromagnetic field. The surface enrichment strategies, including functionalized 89 chemical bonding ${ }^{18,19}$, chemical reaction ${ }^{20}$, physical adsorption ${ }^{21,22}$, optical trapping ${ }^{23}$ as well as 90 passive trapping by undercut structure ${ }^{24}$ bring in the additional working requirements (e.g., 91 chemical stimuli, temperature, pressure, pump power, etc.) for specific molecules, impeding the 
92 system for global molecular recognition. Thus, the performance of the sensing system needs to

93 improve by engineering the PNA structure. The intuitive way is to increase the intensity of the

94 electric field by squeezing the gap between adjacent PNA into nanometer scale. ${ }^{25-27}$ However, the

95 narrow gap ruins the sensing performance by a decrement in the active area and increases

96 fabrication cost. Therefore, other approaches like hybrid 2D materials ${ }^{28-30}$ and

97 homo/heterogeneous bonding ${ }^{31,32}$ are developed to bypass the fabrication using lithography of

98 desired nanogap and to achieve a large area electromagnetic hot spots for sensing. Additionally,

99 the loss engineering method is proposed to tune the coupling condition between PNA and

100 molecular vibration by adjusting the loss of antenna from specific structures to achieve optimized

101 enhancement. ${ }^{32,33}$

102 In addition to sensitivity, the detection range is another critical FOM of PNA sensors, reflecting

103 the number of fingerprint absorption peaks that can be captured. Thanks to the sharp resonance

104 peaks of PNA, the enhancement becomes the maximum only when molecule fingerprint absorption

105 peaks match with the PNA resonance, which is a very narrow bandwidth. Therefore, to detect more

106 absorption peaks in the MIR region, multi-resonant PNA sensors are proposed to collect broadband

107 spectrum data to recognize lipids and proteins from separate absorption wavelengths. ${ }^{34}$

108 Nevertheless, the individual resonances of PNA by a different order of resonance modes also fails

109 to cover the whole spectrum of IR fingerprint wavelength region from $5.5 \mu \mathrm{m}$ to $10 \mu \mathrm{m}$ because

110 of the gaps between two resonance peaks. ${ }^{35,36}$ Therefore, to collect continuous spectral fingerprint

111 absorption, pixelated all-dielectric nanoantenna array and tunable antenna by incident angle were

112 proposed for ultra-broadband spectroscopic analysis for molecular barcode imaging and

113 fingerprint absorption retrieving. ${ }^{37,38}$ However, the data from the different pixels are collected

114 separately, which cannot achieve dynamic monitoring. Hyperspectral IR imaging could be an 
115 advanced solution to solve the problems, and PNA has been used to enhance the vibrational

116 molecular IR imaging captured by the focal plane array (FPA). ${ }^{39}$ Furthermore, spectrum

117 reconstruction by IR imaging technology is reported to retrieve wavelength shift of nanoantenna

118 for molecular identification as refractometry microscopy with one-time data acquisition, but the

119 performance is still limited due to the use of a monochromatic light source. ${ }^{40}$

120 We proposed a novel molecular identification platform by wavelength gradient hook 121 nanoantenna surface-enhanced infrared absorption spectroscopy (WGHNA-SEIRAS) to enhance

122 the sensitivity and detection bandwidth of PNA based spectroscopy by engineering the loss of

123 PNA sensor from hook nanoantenna (HNA) structure based on temporal coupled-mode theory

124 (TCMT) and pixelating wavelength-gradient HNA building blocks. Based on WGHNA-SEIRAS,

125 we proposed new methods to recognize three alcoholic molecules (methanol, ethanol, and 126 isopropyl alcohol) in a mixture as a proof-of-concept demonstration by decoupling the 127 complementary physical (refractive index) and chemical (fingerprint absorption of the chemical 128 bond) information from IR spectrum via principal component analysis (PCA). Furthermore, with 129 the aid of hyperspectral imaging of pixelated HNA array, we reconstructed the enhanced 130 fingerprint absorption spectrum by one-time data acquisition from the aqueous analyte of acetone, 131 isopropyl alcohol (IPA), and their mixture. We also integrated the WGHNA-SEIRAS platform 132 with a microfluidic channel, solving the water absorption issue by traditional IR spectroscopy for 133 aqueous measurement, which can be easily compatible with the biomolecular and cellular systems. 134 Our work paves the way to achieve fast global molecular recognition by rich spectrum information 135 from HNA-SEIRAS in non-contact, non-destructive, label-free, and miniaturized methods. 


\section{Working Principles of WGHNA-SEIRAS}

137 The concept of WGHNA-SEIRAS platform is shown in Fig.1. The MIR light shines from an IR 138 microscope and excites the plasmonic resonance of HNA on calcium difluoride $\left(\mathrm{CaF}_{2}\right)$ substrate with desired polarization state and perpendicular incidence. The transmitted or reflected light is routed to IR FPA to capture the far-field spectral response from HNA. With the plasmon-phonon coupling illustrated in Fig.1 a, the resonant HNA interacts with the molecular vibration at matched wavelength/frequency, thus enhancing the fingerprint absorption. Based on TCMT, we can get equations for coupling system ${ }^{33}$ (Method 1) and derive the transmission and reflection spectral desperation as

$$
T(\omega), R(\omega)=\left|\frac{S_{\mathrm{t}, \mathrm{r}}}{S_{\mathrm{in}}}\right|^{2}=\left|\frac{j\left(\omega-\omega_{0}\right)+\gamma_{a, r}+\frac{\mu^{2}}{j\left(\omega-\omega_{m}\right)+\gamma_{m}}}{j\left(\omega-\omega_{0}\right)+\left(\gamma_{a}+\gamma_{r}\right)+\frac{\mu^{2}}{j\left(\omega-\omega_{m}\right)+\gamma_{m}}}\right|^{2}
$$

where $\omega_{0}$ and $\omega_{m}$ represent the angular frequency of resonance for HNA and molecular vibration, respectively. $\gamma_{a}$ and $\gamma_{r}$ denote the radiative and absorptive losses of HNA, while $\gamma_{m}$ is the absorptive loss of molecules. $\mu$ is the coupling strength between HNA and molecular vibration.

149 From Eq.1, the enhanced vibration signal can be observed as electromagnetic induced transparent 150 (EIT)-like line shape, a noticeable dip in HNA resonance when two resonance modes are well151 matched $\left(\omega_{0}=\omega_{m}\right)$. The substantial enhancement of the sensing signal is observed in the change 152 of transmission or reflection intensity compared with intrinsic molecule absorption.

153 To achieve a broadband response of the HNA platform, we propose two types of wavelength154 gradient design by gradually changing the optical length of HNA. One is the HNA array by a 155 gradient change in each pixel with periodic nanoantenna structures in the same scale, and the other 156 is the HNA supercell by the gradient structure into unit cells for periodic structure, as shown in 157 Fig.1 d. The respective spectrum for HNA array and supercell is demonstrated in Fig. 1 c and e 
with the concept showing of interaction with molecules absorption peaks. The WGHNA shows the wavelength-scalable response to capture multiple fingerprint absorption peaks. With imaging processing and $\mathrm{ML}$ for the raw data, the fingerprint barcoding and molecule identification are achieved for array and supercell, respectively. In Fig. $1 \mathbf{f}$, the barcoding of molecular absorption peak is demonstrated for broadband fingerprint retrieval. The blue and green colors indicate the existence of the chemical bond, and the brightness of each pixel indicates the absorptance corresponding to the concentration of one type of chemical bond. Each pixel of the HNA array only covers a limited bandwidth near the resonance wavelengths, which gives the highest sensitivity for plasmon phonon coupling. Besides, the PCA is used to process the broadband signal from the HNA supercell to classify different types of molecules. The function of PCA is to amplify the difference of molecular spectra with massive raw data collected. As demonstrated in Fig. $1 \mathbf{~ g}$, three clusters can be observed to recognize three different molecules.

\section{Design and Sensing Characterization of HNA}

The design concept and experimental results of HNAs are shown in Fig.2. From equation 5, we observe that the $\mathrm{T}$ and $\mathrm{R}$ are related to the resonance properties of HNA, which are radiative and absorptive losses $\left(\gamma_{a}\right.$ and $\left.\gamma_{r}\right)$. The $\gamma_{a}$ is related to the ohmic loss of plasmonic material (e.g., Au in this work) and is almost robust among different antenna structures. Therefore, the philosophy to use hook shape in PNA design is to engineer $\gamma_{r}$ to tune the radiation from electron oscillation by inducing inverse current from short arm (L3) of HNA. The method to control radiation capability from the ratio of inverse current is merely adjusting the geometric difference $(\Delta \mathrm{L})$ between long arm (L1) and short arm (L3) of HNA as illustrated in Fig.2 a. The HNA performs a dipole resonance at resonance frequency by enhancing localized electric field intensity, thus inducing the current from one end to the other end (Fig.2 b,c). The connection of two arms of HNA (L2) only affects the resonance wavelength and is defined as a fixed value $(400 \mathrm{~nm})$ to fit the fabrication 
resolution as shown in the SEM photo in Fig. 2 d. With the decrease of $\Delta \mathrm{L}$, both $\mathrm{T}($ Fig.2 e) and R (Fig.2 f) intensities drop, which means the antenna becomes less radiative (darker). However, the absorption signal reaches a peak value when $\Delta \mathrm{L}$ equals $0.6 \mu \mathrm{m}$, which means the critical coupled point $\left(\gamma_{a}=\gamma_{r}\right)$ of the HNA resonator. The experiment results of resonance intensity prove the FDTD simulation observation in Fig. $\mathbf{2}$ h-j.

To characterize the sensing performance of each hook antenna devices, $10 \mathrm{~nm}$ Poly(methyl methacrylate) (PMMA) thin film is coated on top of HNA sensors. A strong resonance of the " $\mathrm{C}=\mathrm{O} "$ stretching is observed at $\sim 5.8 \mu \mathrm{m}$ in Fig. 3. According to TCMT results in Eq. 1, the sensitivity of plasmonic sensors is defined as the intensity change of resonance spectrum of transmission $(\Delta \mathrm{T})$ or reflection $(\Delta \mathrm{R})$ and is expressed as

$$
\begin{aligned}
& \Delta T\left(\omega=\omega_{0}\right)=T\left(\omega=\omega_{0}\right)-\left.T\right|_{\mu=0}\left(\omega=\omega_{0}\right)=\frac{2 \mu^{2}}{\gamma_{a} \gamma_{m}} \frac{f}{(1+f)^{3}} \\
& \Delta R\left(\omega=\omega_{0}\right)=R\left(\omega=\omega_{0}\right)-\left.R\right|_{\mu=0}\left(\omega=\omega_{0}\right)=-\frac{2 \mu^{2}}{\gamma_{a} \gamma_{m}} \frac{f^{2}}{(1+f)^{3}}
\end{aligned}
$$

where $\mu$ and $f$ denote coupling efficiency between HNA and molecular vibration as well as the ratio $\left(\gamma_{r} / \gamma_{a}\right)$ between radiative $\left(\gamma_{a}\right)$ and absorptive $\left(\gamma_{r}\right)$ damping rate of the HNA, respectively. As $\Delta \mathrm{L}$ decreases, $\mu$ remains almost unchanged because of the similar intensity of near field (Supplementary), but $f$ decreases due to the reduced $\gamma_{r}$ caused by the short electrical length of the antenna and the similar $\gamma_{a}$ caused by the same antenna length $(\mathrm{L}=\mathrm{L} 1+\mathrm{L} 2+\mathrm{L} 3)$, as shown in Fig. 3 c. The experimental results of PMMA sensing are shown in Fig. $\mathbf{3}$ a,b, and the extracted difference signals are plotted in Fig. 3 d,e. From Fig.3 f, we observe that the highest sensitivity of transmission mode $(\Delta \mathrm{T})$ comes when $\Delta \mathrm{L}$ equals $0.6 \mu \mathrm{m}$, while $1.2 \mu \mathrm{m}$ (inset SEM for real HNA device) for reflection mode $(\Delta \mathrm{R})$, which agrees with the theoretical prediction from Eq. 6,7 $(f=0.5$ for $\mathrm{T}$ and $f=2$ for R). In Fig. $\mathbf{3} \mathbf{g , h}$, a transition of line shape from Fano-like to EIT-like is observed when the resonance wavelength of HNA matching with molecular absorption wavelengths. 
205 Besides, the highest sensitivity is achieved when the resonance wavelengths of HNA and molecules are well-matched (Fig.3 i). After optimization, the arm length ratio is fixed at L $3: \mathrm{L} 1=1: 3$ to achieve the highest sensitivity at reflection mode. Therefore, to simultaneously achieve the best

208

209

210

211

212

213

214

215

216

217

218

219

220

221

222

223

224

225

226

227

228

sensitivity and broad bandwidth, the wavelength-gradient structures are designed by gradually increasing the total length with the fixed folding degree of HNA.

\section{Characterization of HNA Supercell and Fluidic Dynamics}

The spectrum of the 16-element HNA supercell is shown in Fig.4 a measured by a Fouriertransform infrared (FTIR) spectroscopic microscope (Methods 4). The wavelength gradient response is observed from $\sim 5 \mu \mathrm{m}$ to $\sim 7.8 \mu \mathrm{m}$ with 16 HNA structures by changing the total length (L). To compare the sensing performance of WGHNA, two types of molecules (silk protein and PMMA) are separately coated on HNA supercell. Fig.4 b shows the sensing results of silk and PMMA on HNA supercell with the broadband response from $\sim 5.5 \mu \mathrm{m}$ to $8.5 \mu \mathrm{m}$. Multiple fingerprint absorption peaks are captured by the broadband device. The redshift of the HNA supercell spectrum is caused by the effect induced by the refractive index of analytes indicating the refractometry function of WGHNA. We further compared the sensing performance with selected HNA elements (P1, P8, and P16) from WGHNA, showing that WGHNA has a better enhancement effect of multiple absorption peaks from broad wavelength ranges, while HNA only reaches the best enhancement at narrow wavelength ranges near resonance wavelengths. Both WGHNA and HNA show the significant enhancement ( 3 orders of magnitude) of absorption spectrum with the direct measurement of thin-film without nanoantenna.

In addition to the proof-of-concept characteristic of thin-film, we also integrated WGHNA into a microfluidic system (Fig. 4 d I), which is easily compatible with biomolecular systems for molecular detection in the aqueous environment. The response of HNA supercell for water with the enhancement of $\mathrm{OH}$ bond absorption at $6.0 \mu \mathrm{m}$ is shown in Fig.4 d II. We also performed the 
dynamic monitoring of acetone in water to mimic the real-time dynamic monitoring of metabolic in biology samples. As shown in Fig. $\mathbf{4}$ e, the real-time spectrum indicates the analyte change at the WGHNA surface as time goes by. Multiple fingerprint absorption peaks are captured to have rich information of chemical bond changes. (Fig. 4 f) By integrating the absorbance spectrum, the dynamic behavior of water and acetone can be monitored by the change of chemical bonds. The reduction of $\mathrm{O}-\mathrm{H}$ bond absorptance at $2.95 \mu \mathrm{m}$ and $6.0 \mu \mathrm{m}$ represents the decrease of water concentration, while the increasing of $\mathrm{C}=\mathrm{O}, \mathrm{C}-\mathrm{H}, \mathrm{C}-\mathrm{C}-\mathrm{C}$ bond absorptance at $5.7 \mu \mathrm{m}, 7.0$ and 7.3 $\mu \mathrm{m}, 8.3 \mu \mathrm{m}$ indicate the introduction of acetone molecules into the microfluidic system.

\section{Machine Learning for Molecular Identification}

To demonstrate the molecular identification properties of WGHNA, we select three types of chemically similar alcoholic liquid - methanol, ethanol, and IPA. Both of the molecules have the same functional group of hydroxy and methyl bond, resulting in similar absorption spectra in $6 \mu \mathrm{m}$ to $9 \mu \mathrm{m}$ wavelengths. Therefore, it is not easy to distinguish them in a mixture with a narrow bandwidth of HNA. We designed a series of experiments to compare the recognition capability of HNA and WGHNA using 1\% methanol, ethanol, and IPA in water and mixture sets of each two in the same volume. With the injection of liquid from microfluidics, the response of HNA and WGHNA is plotted in Fig. 5 a. The apparent dips of the reflection spectrum at $6.0 \mu \mathrm{m}$ are induced by water in both HNA and HNA supercell. The fingerprint absorption of alcoholic molecules is captured from $6.5 \mu \mathrm{m}$ to $9 \mu \mathrm{m}$ and is extracted from the HNA supercell spectrum in Fig. 5 b. Due to the low concentration of analytes, the change of reflection at absorption is small and cannot be detected without HNA. To process the small signal, we applied a second derivative to extract the characteristic of each spectrum from the HNA supercell (Fig. 5 c), which is widely used in traditional IR spectroscopy analysis. However, it is still difficult to distinguish clearly with the classic data processing methods from the enhanced spectrum of HNA by solely analyzing the 
253 fingerprint absorption. Therefore, we propose a ML method using PCA to process the HNA data

254 for extraction of multi-dimensional information from HNA, which is absorption peaks induced by 255 vibration of the chemical bond, the wavelength shift of HNA resonance induced by the refractive 256 index of molecules, and the intensity change of water absorption induced by loading effect of 257 wavelength detuning.

258 The results of PCA processed spectra are shown in Fig. $5 \mathbf{d}$ by dimension reduction to three 259 principal components (PC) axes. For HNA spectra, the first PC represents the modulation of water 260 absorption peak by loading effect of wavelength shift, and the second PC represents the 261 wavelength shift of HNA resonance induced by refractive index if analytes. While for HNA 262 supercell, the first and second PC is flipped in terms of data feature from the spectrum. The third 263 PC represents the fingerprint absorption of three molecules in both the HNA and HNA supercell. 264 The order of PC represents the degree of difference between each spectrum. In 3D PC space, each 265 point represents the spectrum data from HNA or HNA supercell, and each cluster represents one 266 type of molecule combination. In conclusion, with the help of PCA, the IR spectrum of HNA with 267 different molecules can be reduced to three principal components, which indicating the three key 268 features of loading effect, wavelength shifts, and enhanced fingerprint absorption. With the full 269 utilization of multiple dimension information, the recognition becomes more efficient by 270 monitoring the complementary physical (refractive index) and chemical properties (absorption 271 fingerprints) of molecules, bring in a new degree of freedom into IR spectroscopy analysis by 272 refractometry and plasmonic properties. Compared with previous literature that demonstrates the 273 identification of two molecules mixture by monitoring two absorption peaks ${ }^{17}$, our work 274 demonstrated simultaneously monitoring of 15 absorption peaks and used to identify three 275 molecules mixture. Furthermore, with the aid of dimension reduction by PCA, the multi- 
276 dimensional information from HNA is easily decoupled and analyzed, paving the way to achieve

277 global molecular identification and real-time monitoring by training with deep neuron networks

278 (DNN).

279 Hyperspectral Imaging of HNA Array for Fingerprint Reconstruction

280 Hyperspectral imaging is applied to the HNA array to retrieve the enhanced fingerprint absorption

281 with one-time data acquisition. As shown in Fig. 6 a-d, the $4 * 4$ HNA array (P1-P16) with

282 wavelength gradient is used to capture the hyperspectral image from $4 \mu \mathrm{m}\left(2500 \mathrm{~cm}^{-1}\right)$ to $9 \mu \mathrm{m}$

$283\left(1111 \mathrm{~cm}^{-1}\right)$ by FPA under different analyte states (Bare, acetone, IPA and mixture). The

284 fingerprint absorption of acetone and IPA is reflected on the hyperspectral image of HNA array at

285 absorption wavelengths. In the mixture of Acetone and IPA, the combination of image change is

286 observed at all absorption wavelengths. To get a better understanding of the spectral response of

287 the HNA array, we extract the spectra of each HNA pixel in Fig. 6 e I-III and further calculate the

288 difference of reflection signal induced by molecular absorption in Fig. 6 e IV-VI. By integrating

289 the molecular spectra within a fixed bandwidth of each pixel $(400 \mathrm{~nm})$, we reconstructed the

290 fingerprint absorption barcode of Acetone, IPA, and their mixture in Fig. 6 f. The darker the color

291 of each pixel leads to the strong absorption induced by molecules. It agrees well with the

292 fingerprint absorption of IPA and Acetone captured by traditional IR spectroscopy. The absorption

293 peaks of IPA at 7.0 to $8.0 \mu \mathrm{m}$ and $8.5 \mu \mathrm{m}$ are captured by P9 to P13 and P16 (follow same

294 orientation in Fig. 6 a) respectively, while the absorption peaks of acetone at $5.74 \mu \mathrm{m}, 7.0$ to 7.5

$295 \mu \mathrm{m}$ and $8.0 \mu \mathrm{m}$ are captured by P1 to P3, P9 to P11 and P14 respectively. In the mixture sample,

296 both absorption peaks are captures from the reconstructed imaging. The retrieved fingerprint

297 absorption barcode of the mixture shows a combination of the barcode image between IPA and

298 Acetone. Such rich spectral information is captured in one-time data acquisition, and the IR

299 fingerprint absorption is enhanced by the HNA array. Compared with the similar approach 
300 achieved by all-dielectric nanoantenna with high quality factors ${ }^{37,38}$, our approach behaves smaller 301 footprint and spatial tunability so that the whole enhanced spectra can be captured in one testing, 302 which dramatically reduced the time for broadband fingerprint retrieving, paving the way to 303 ultrasensitive and ultrafast molecules screening in ultra-broadband wavelength range with ultra304 small volume. Although the spatial resolution is limited by the pixel of FPA (32*32 pixels) in our 305 demonstration ( $4 * 4$ HNA pixels) to avoid mutual coupling, it is easy to improve by replacing the 306 FPA with more pixel numbers, smaller pixel area, and better detectivity (D*).

\section{Discussion}

In this work, we propose a novel WGHNA-SEIRAS with high sensitivity and broad bandwidth

309 for molecular identification with complementary information of physical (refractive index) and 310 chemical (chemical bond fingerprint absorption) properties. With a demonstration of thin-film 311 sensing of PMMA and silk protein, and microfluidic sensing of water, acetone, and acholic 312 molecules, the WGHNA show the enormous potential of real-time broadband dynamic detection 313 of the molecular behavior like chemical reaction and various molecular recognition. Additionally, 314 with the aid of machine learning algorithm-PCA, the multi-dimensional rich information can be 315 classified effectively, resulting in good pattern recognition using both spectroscopy and 316 refractometry function from WGHNA-SEIRAS. Furthermore, leveraging FPA for hyperspectral 317 imaging of WGHNA, the enhanced fingerprint absorption with rich information of multiple 318 molecular vibrational peaks from broad bandwidth (from $4 \mu \mathrm{m}$ or $2500 \mathrm{~cm}^{-1}$ to $9 \mu \mathrm{m}$ or $1111 \mathrm{~cm}^{-}$

$31{ }^{1}$ ) can be captured in one-time data acquisition, booting up the screening speed and recognition 320 capability for global molecule identification. Our work brings new insights into IR spectroscopy 321 technologies for small-volume, real-time, ultra-sensitive, in-vitro biomolecular dynamic analysis 322 in the aqueous environment. 

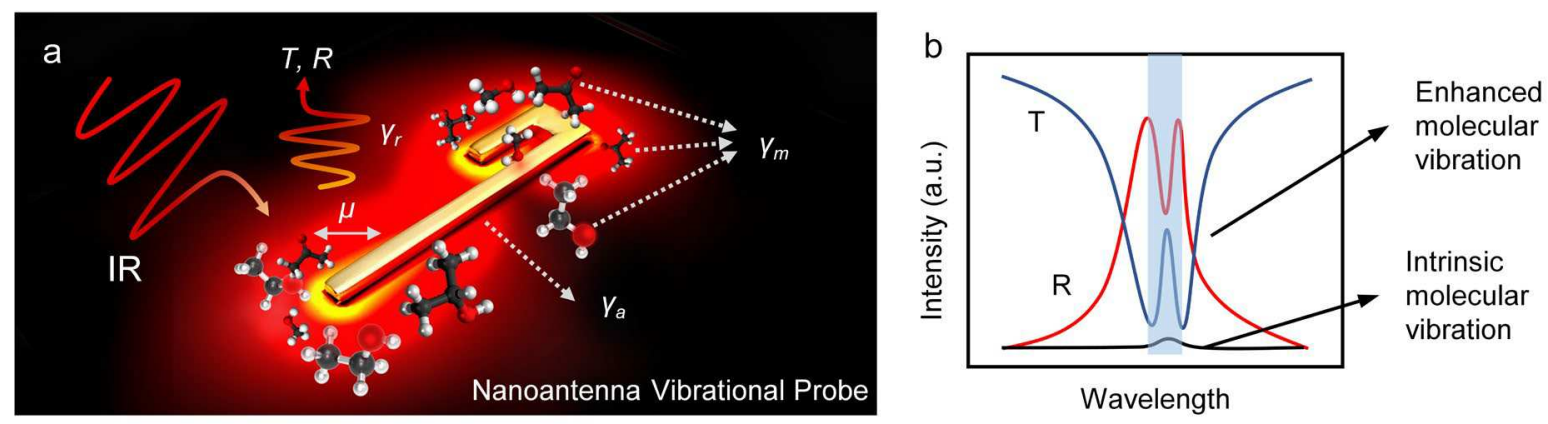

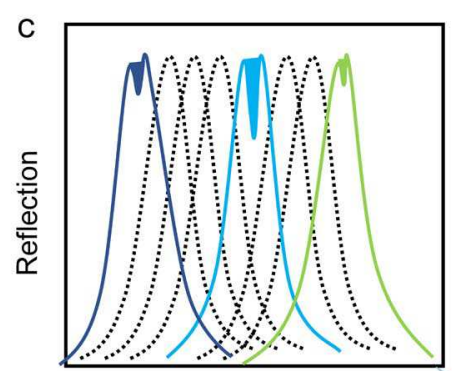

Wavelength

Image processing

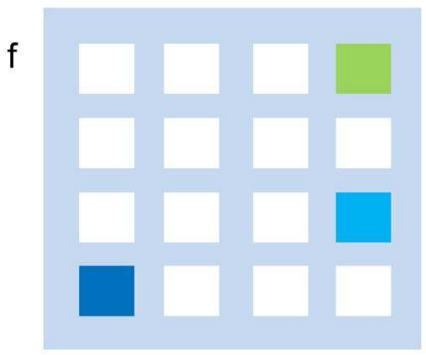

Fingerprint bar-coding
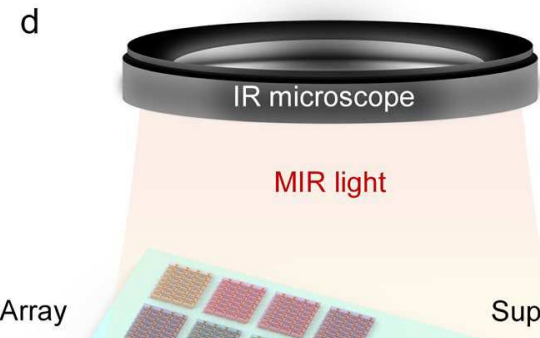

MIR light

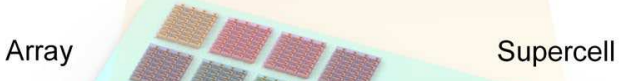

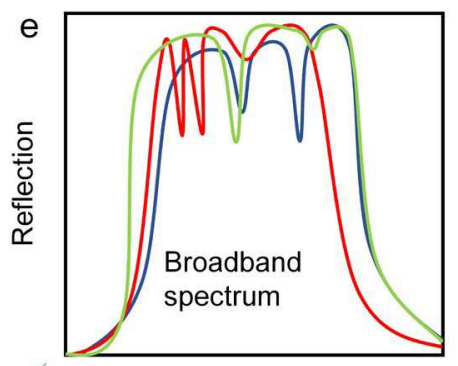

Wavelength

Machine learning

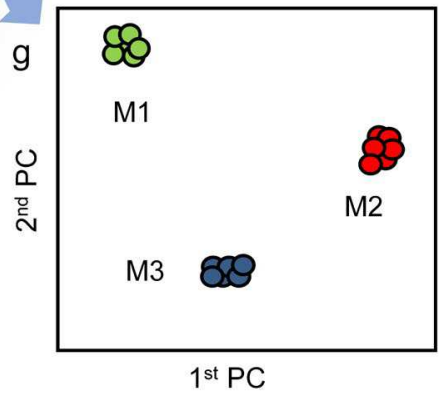

Fig.1 (a) Schematic drawing of HNA vibrational probe for molecular sensing by the interaction between plasmonic resonance and molecular vibration. The yellow color refers to the simulated electrical field near the surface of the hook antenna. The physical parameters $\left(\gamma_{a}, \gamma_{r}, \gamma_{m}\right.$ and $\left.\mu\right)$ are used in TCMT modeling to express the resonance behavior of PNA and molecular vibration. (b) The spectrum of plasmonic-enhanced molecular vibration signal in reflection $(R)$ and transmission (T) compared with intrinsic fingerprint absorption. (c) The concept drawing of spectral response of wavelength-gradient HNA array with molecular vibration fingerprints. The different curves indicate the spectrum of each pixel in the HNA array. Each HNA pixel has one periodic HNA structure and the length of HNA gradually changes among different pixels. By gradually increasing the optical length of HNA in one pixel, the optical resonant wavelength is also increasing linearly. The molecular vibration is captured by the HNA pixel which operates at the same wavelength. The enhanced absorption is marked as shadow by different color for different pixels. (d) Schematic drawing of two types of WGHNA designs - HNA array and HNA supercell. Both HNA array and supercell have the wavelength gradient nanoantenna structure. The difference is that the wavelength gradient is designed at different order of structures, which are pixel and cell levels. The HNA array has periodic HNA structure at each pixel and changes among different pixels, while the HNA supercell holds the gradient nanoantenna into one unit-cell called 
supercell and repeat the supercell to form periodic structures. The inset SEM image is the topview of HNA, and the scale bar indicates $1 \mu \mathrm{m}$. (e) The concept drawing of spectral response of wavelength-gradient HNA supercell with molecular vibration fingerprints. Example illustration of different molecular vibration fingerprints are marked in of blue, green, and red. The broadband response of supercell shows the capability to capture multiple absorption peaks. (f) The fingerprint barcoding image is processed from the HNA array pixel signal in (c). (g) Molecular recognition results from the broadband spectrum of HNA using principal component analysis (PCA) algorithm from machine learning. Each cluster indicates one type of molecule.
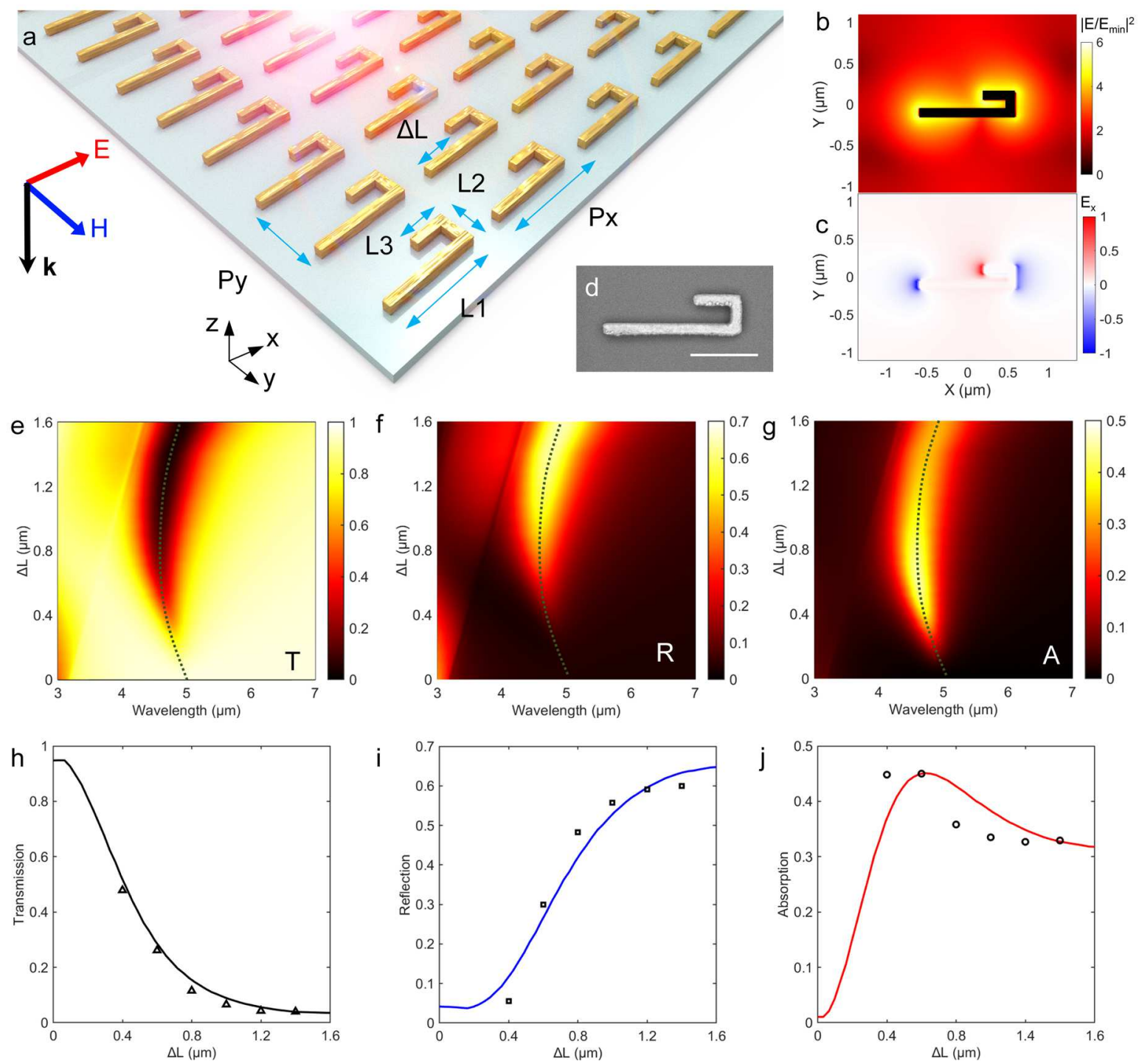

Fig.2 The plasmonic properties of HNA. (a) The schematic drawing of the HNA array. The polarization of incident light is aligned with the long arm of HNA. $\triangle L$ is used to characterize the folding degree of the hook antenna. (b) Simulated nearfield distribution of electric field intensity of $H N A$ when $\Delta L$ equals $1.2 \mu \mathrm{m}$. (c) Simulated nearfield distribution of electric field polarity of $H N A$ when $\Delta L$ equals $1.2 \mu \mathrm{m}$. The dipole resonance is generated at the resonance wavelength. (d)The SEM image of one HNA with scale bar indicating $500 \mathrm{~nm}$. (e-g) the IR response spectrum as the increment of $\Delta L$ for transmission (e), reflection (f), and absorption ( $g$ ). (h-i) The experimental results (points) as the increment of $\Delta L$ compared with simulation results (curves). 

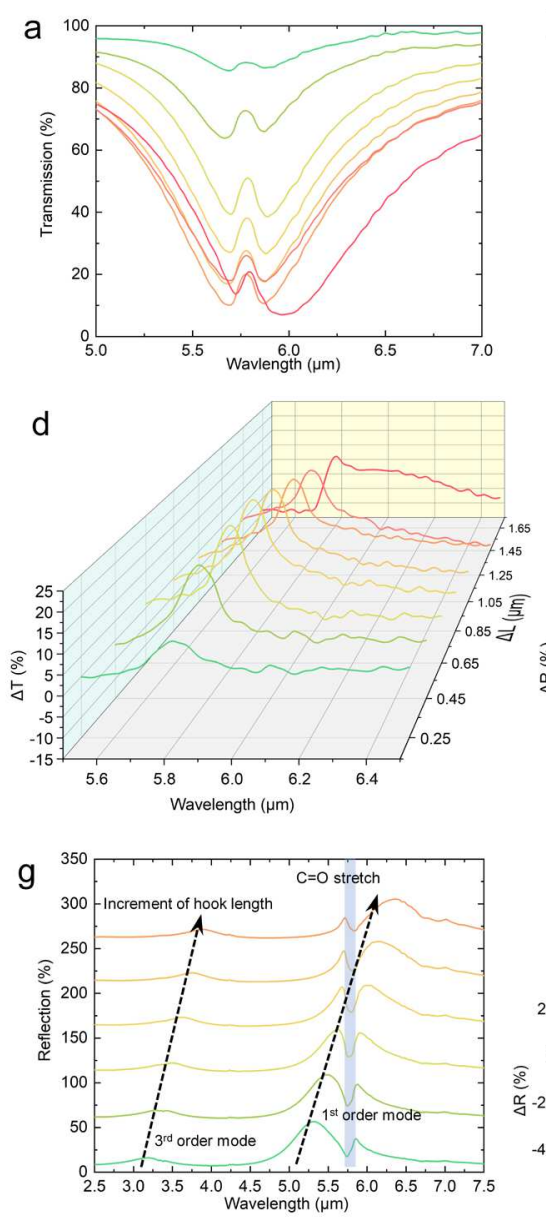
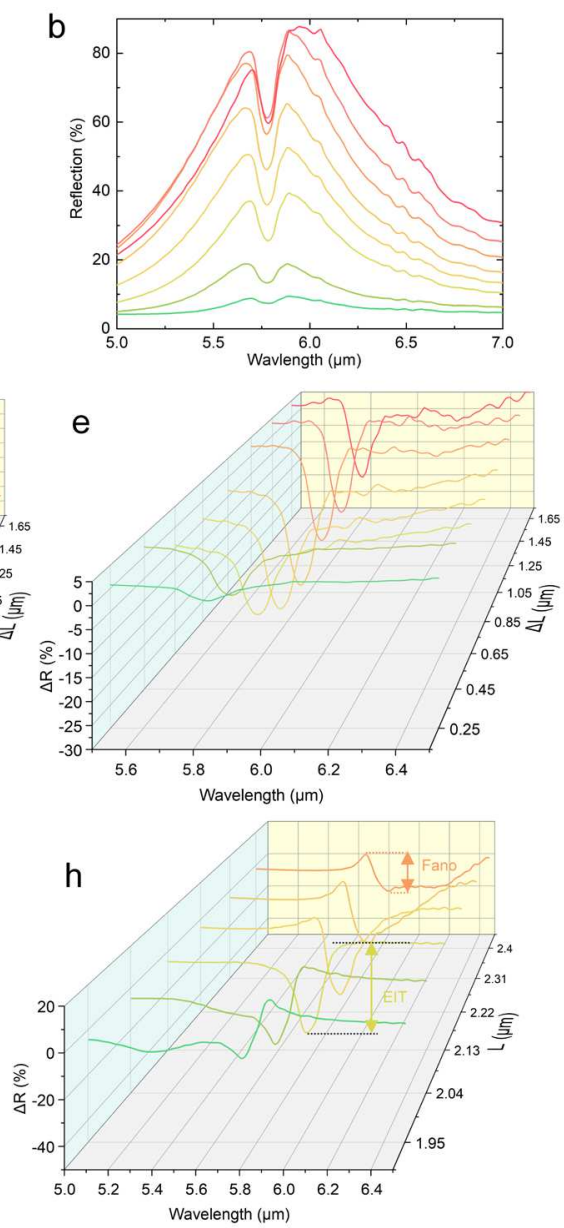
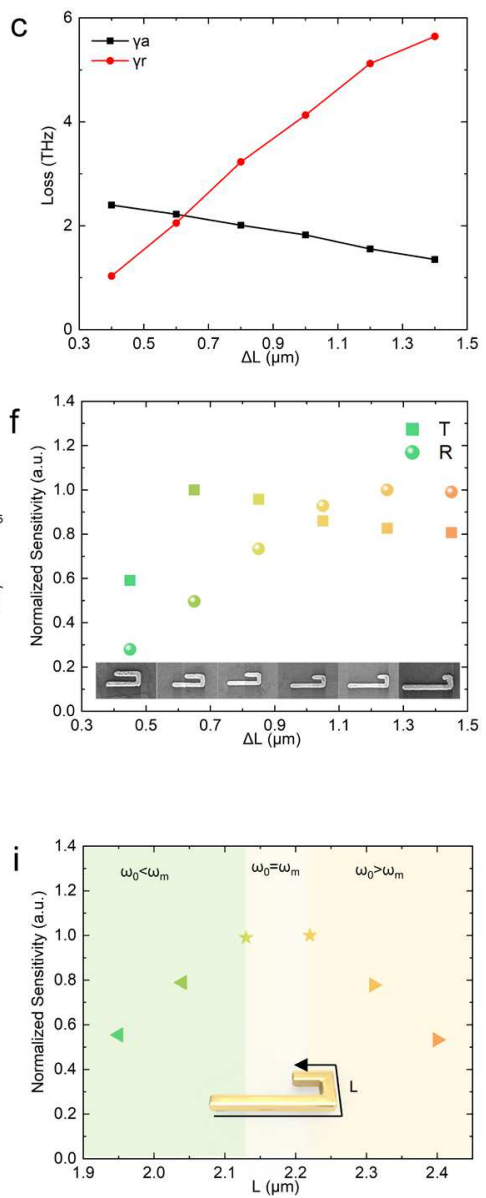

Fig.3 Characterization of sensing performance for HNA. (a,b) Experimental sensing spectrum of $H N A$ with variances of $\triangle L$ for $C=O$ stretch of PMMA. (c) Theoretical fitting of damping rate of a different folded degree from TCMT. (d-e) Extracted sensing signal of HNA with a difference with reference devices. (e)the sensitivity of the HNA sensor of transmission and reflection mode with the change of $\Delta L$. (g) experimental sensing spectrum of $H N A$ with variances of $L$ for $C=O$ stretch of PMMA. (h) Extracted sensing signal of HNA with a difference with reference devices. (i) the sensitivity of the HNA sensor of transmission and reflection mode with a change of $L$. 

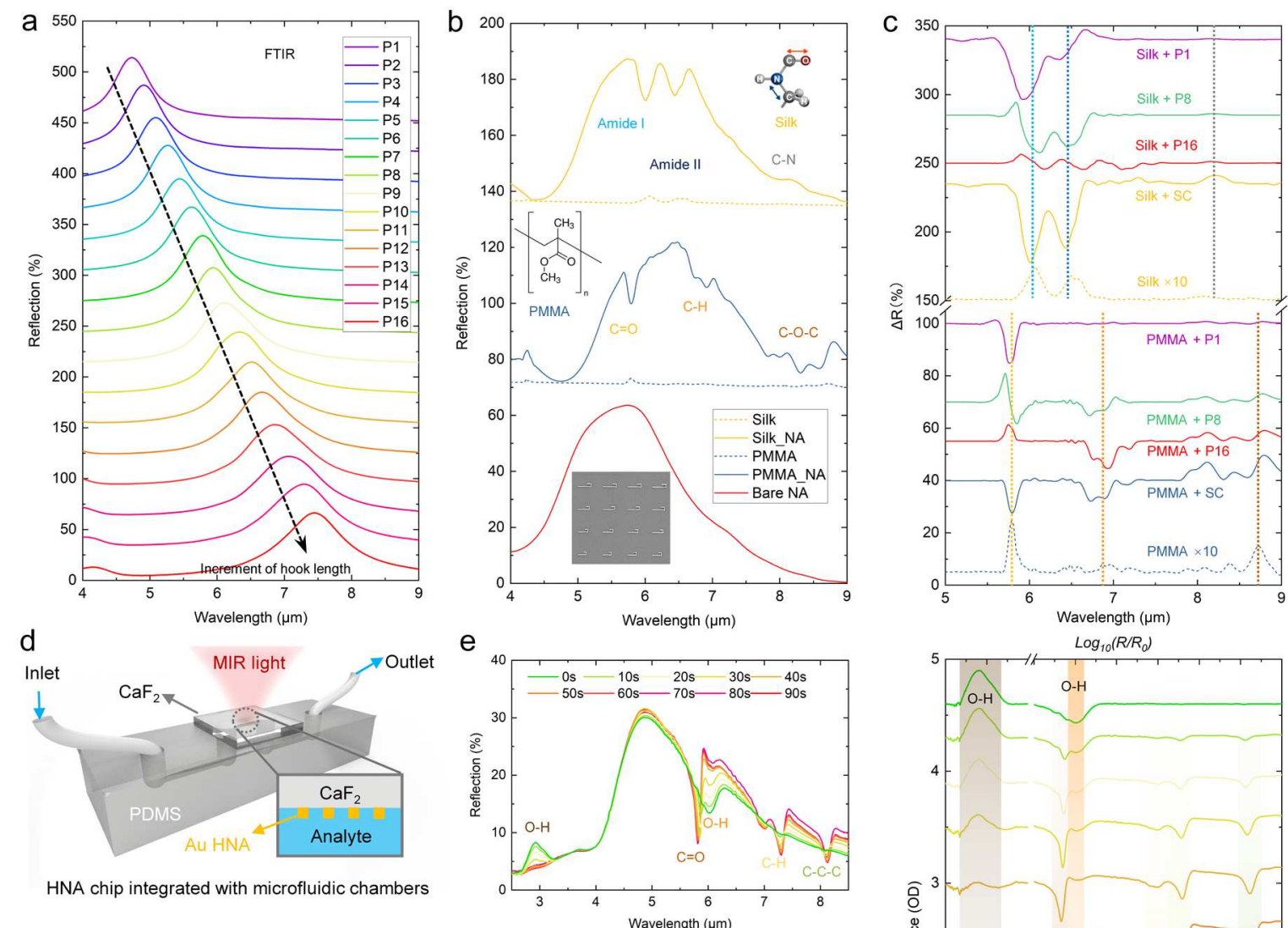

HNA chip integrated with microfluidic chambers
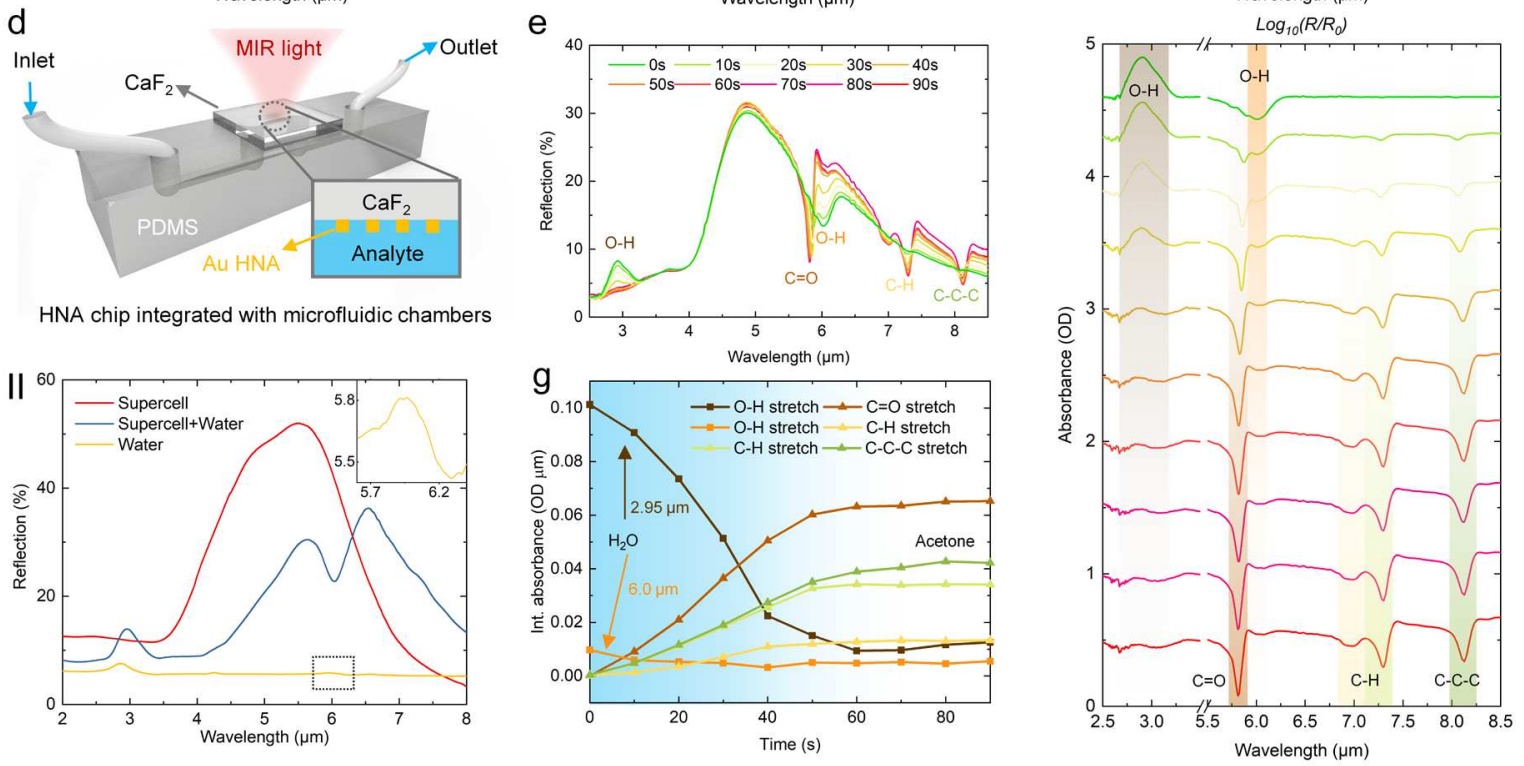

Fig.4 Broadband sensing characterization of HNA supercell by gradient increase HNA length into a unit cell and broadband monitoring of fluidic dynamics for acetone injection into the water from HNA supercell integrated with PDMS microfluidic chamber. (a) The experimental reflection spectrum of HNA by changing the length. A longer HNA leads to a longer resonant wavelength. By combing different lengths into a unit cell of the metasurface, and HNA supercell is formed with broadband resonance performance. (b)Sensing characterization of HNA supercell with the thinfilm analyte of PMMA and silk protein. The fingerprint absorption peaks ranging from $5.5 \mu m$ to $9 \mu \mathrm{m}$ are clearly captured by $\mathrm{HNA}$ supercell including $\mathrm{C}=\mathrm{O}, \mathrm{C}-\mathrm{H}, \mathrm{C}-\mathrm{O}-\mathrm{C}$ bond from PMMA and amide I, amide II and $C-N$ bond from silk. (c)Comparison of sensing spectrum of PMMA and silk between HNA and HNA supercell. The HNA from P1, P8, and P16 is selected as a reference with the response to short, medium, and long-wavelength resonance, respectively. It shows $H N A$ supercell has a good response over a broad range of wavelengths from $5.5 \mu \mathrm{m}$ to $9 \mu \mathrm{m}$ which HNA 
only covers a narrow bandwidth near resonance wavelength for enhancement of fingerprint absorption. (d)I. Schematic drawing of an integrated microfluidic HNA supercell system for liquid sensing. The HNAS on the CaF 2 carrier chip is flip bonded to the PDMS surface with the alignment of HNAS into the microfluidic channel. The microfluidic channel is formed by a $3 D$ printed mold and is fixed on a microscope slide. The IR light is shining from the backside of the CaF 2 chip, and reflected light is collected to monitor the far-field response of HNA supercell with different aqueous analytes. II. The response of $\mathrm{HNAS}$ under the water $\left(\mathrm{H}_{2} \mathrm{O}\right)$ environment. The enhancement of the $\mathrm{O}-\mathrm{H}$ bond of $\mathrm{H}_{2} \mathrm{O}$ molecules at $6.0 \mu \mathrm{m}$ is observed at an enhancement factor of. Additionally, the redshift of um from HNAS is observed to demonstrate the refractometry function of HNAS corresponding to the refractive index change of molecules. (e) The dynamic response of HNAS with respect to a different time as acetone injects into water. Each curve indicates the real-time spectrum of a mixed solvent of acetone and water, reflecting in-situ concentration information of acetone and water and dynamic change versus time. (f). Baseline-corrected absorbance spectrum at a different time at board wavelengths range from 2.5 to $3.5 \mu \mathrm{m}$ for $\mathrm{O}-\mathrm{H}$ bond of water at 2.95 $\mu \mathrm{m}$ and 5.5 to $9 \mu \mathrm{m}$ for various fingerprint peaks ( $\mathrm{O}-\mathrm{H}$ bond for $\mathrm{H}_{2} \mathrm{O}$ at $6.0 \mu \mathrm{m}, \mathrm{C}=\mathrm{O}, \mathrm{C}-\mathrm{H}, \mathrm{C}-\mathrm{C}$ $C$ bond for acetone at $5.7 \mu \mathrm{m}, 7.0$ and $7.3 \mu \mathrm{m}, 8.3 \mu \mathrm{m}$, respectively). (g). The integrated absorbance of each fingerprint absorption as a function of time. As time goes by, the absorbance of O-H bond of $\mathrm{H}_{2} \mathrm{O}$ at $2.95 \mu \mathrm{m}$ and $6.0 \mu \mathrm{m}$ decrease, indicating the concentration decrease of water molecules while the absorbance of $\mathrm{C}=\mathrm{O}, \mathrm{C}-\mathrm{H}, \mathrm{C}-\mathrm{C}$ - $\mathrm{C}$ bond for acetone at $5.7 \mu \mathrm{m}, 7.0$ and $7.3 \mu \mathrm{m}, 8.3 \mu \mathrm{m}$ increase, representing the concentration increase of acetone molecules. 


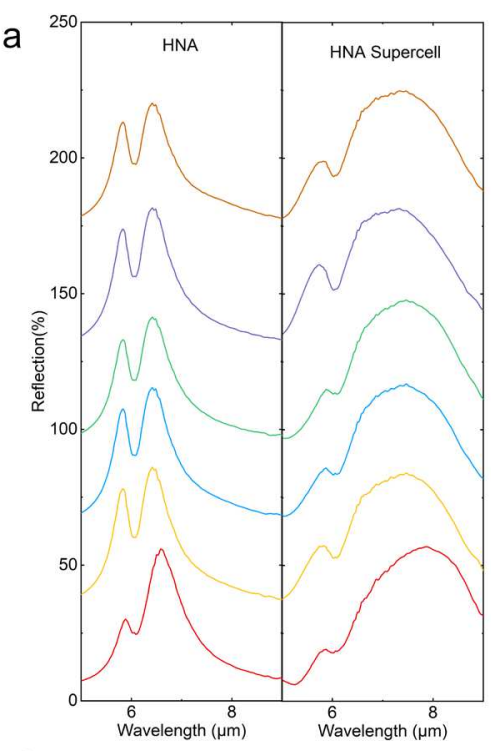

d

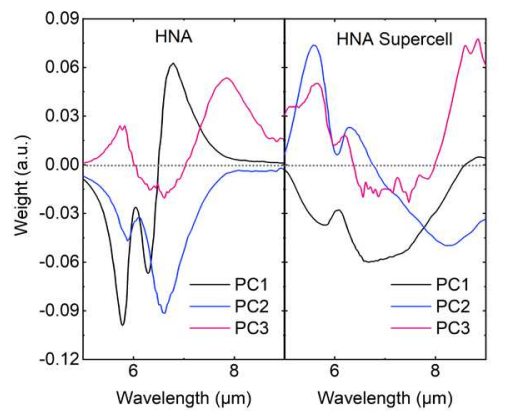

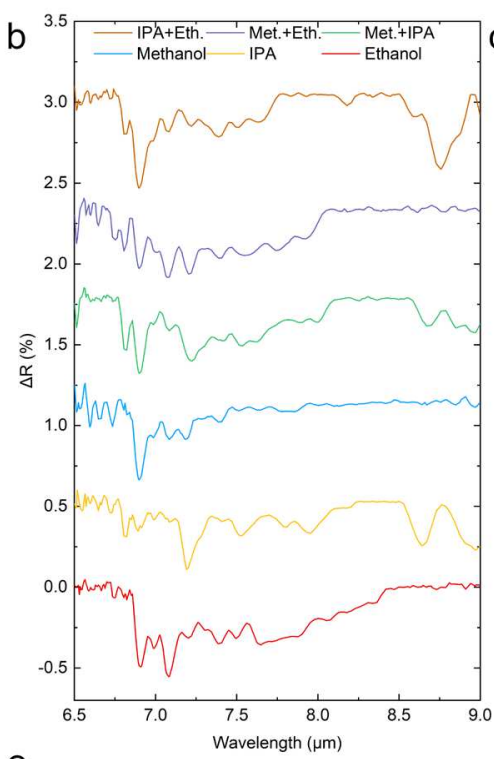

e

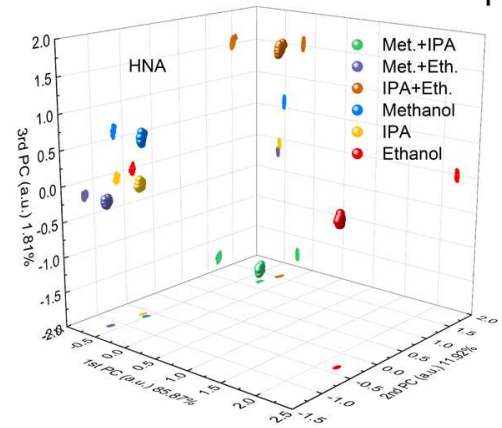

$f$
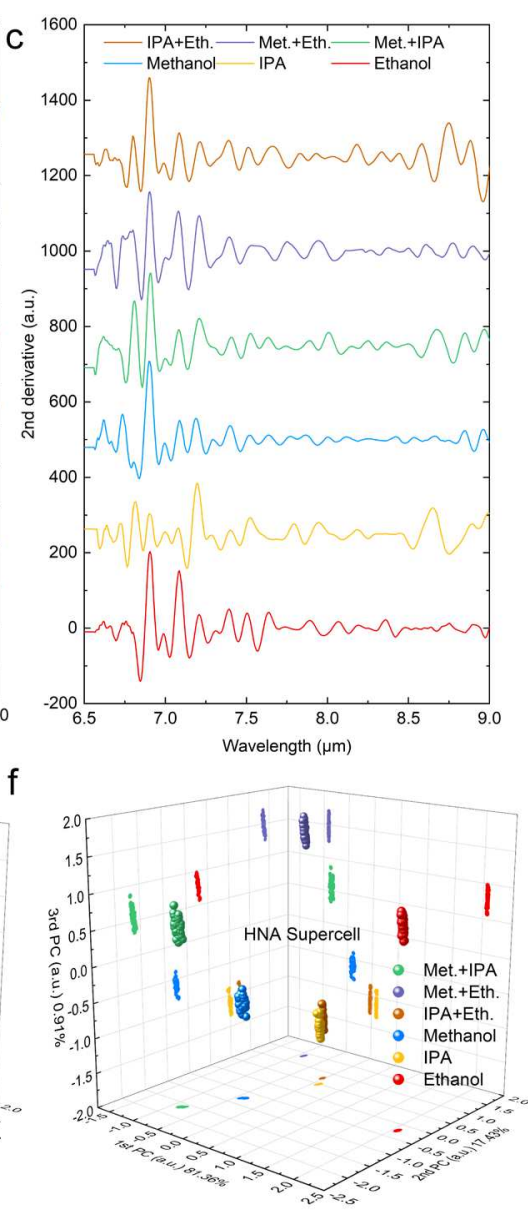

400

401

402

403

404

405

406

407

408

409

410

411

412

413

414

415

416

417

Fig. 5 Machine learning demonstration of HNA spectroscopy by recognition of alcoholic molecules of methanol $\left(\mathrm{CH}_{3} \mathrm{OH}\right)$, ethanol $\left(\mathrm{C}_{2} \mathrm{H}_{5} \mathrm{OH}\right)$, and $\mathrm{IPA}\left(\mathrm{C}_{3} \mathrm{H}_{7} \mathrm{OH}\right)$ at a concentration of $1 \%$ in water $\left(\mathrm{H}_{2} \mathrm{O}\right)$. (a) The response of HNA spectroscopy under different types of alcoholic molecules. The HNA only response to a narrow bandwidth near resonance wavelength at $\sim 6.5 \mu \mathrm{m}$, while HNA supercell response to a wide bandwidth from $6 \mu \mathrm{m}$ to $9 \mu \mathrm{m}$. The dip at $\sim 6.0 \mu \mathrm{m}$ represents the $O$ $H$ bond of water, which is the common solvent in both cases. (b) The calculated the reflection change spectrum (i) and its second derivative (ii) of HNA supercell under different types of alcoholic molecules from $6.5 \mu \mathrm{m}$ to $9 \mu \mathrm{m}$, showing the fingerprint absorption peaks of each molecule and in a mixture of two types of molecules. (c) The machine learning processed a spectrum of HNA spectroscopy after dimension reduction by principal component analysis. For $H N A$, the $1^{\text {st }}$ principle component represents the modulation effect of water absorption peaks at $6.0 \mu \mathrm{m}$. The $2^{\text {nd }}$ principle component represents the wavelength shift of HNA due to the refractive index of the analyte. The $3^{\text {rd }}$ principle component represents the fingerprint absorption of molecules. While for HNA supercell, the $1^{\text {st }}$ and $2^{\text {nd }}$ component is flipped as the HNA case indicating the different response for refractometry and spectroscopy function. (d) The weight of scores of each spectrum in three-dimensional space after PCA for HNA(i) and HNA supercell(ii). Each cluster indicates one type of molecules and their mixtures. 

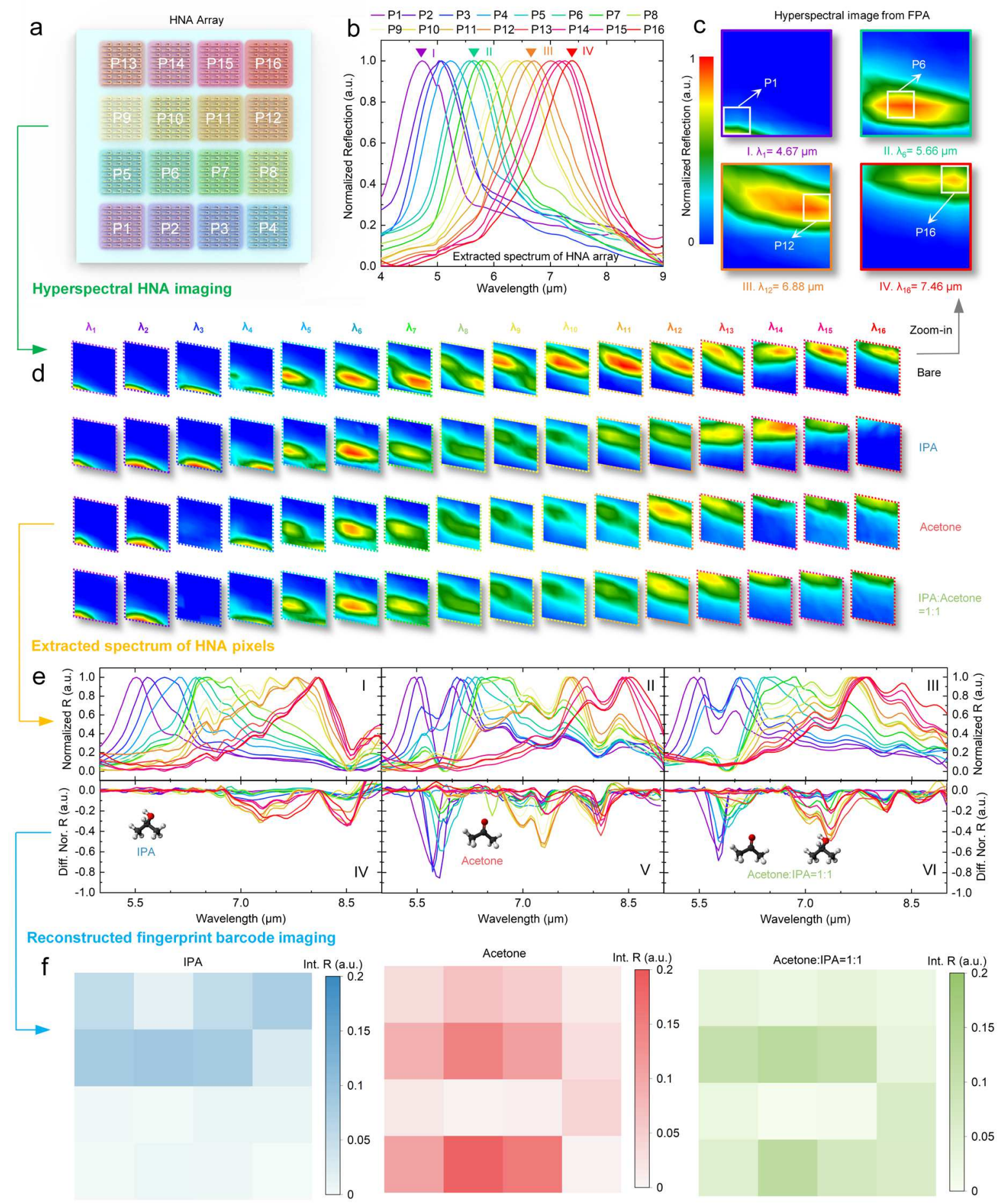

Fig.6 IR fingerprint retrieval and molecules identification by hyperspectral IR imaging for HNA array. (a) Schematic illustration of wavelength gradient HNA array for hyperspectral imaging. 421 Each pixel response to different IR wavelengths. (b) By pixelating the wavelength gradient HNA 422 into a four by four arrangement, the hyperspectral imaging is captured by the FPA, representing 423 the different spectrum response of each HNA pixels. P1 response shortest wavelengths $(\sim 4.67 \mu \mathrm{m})$ 424 and P16 response the longest wavelengths $(\sim 7.46 \mu \mathrm{m})$. The wavelength difference is designed to 
425 be $\sim 200 \mathrm{~nm}$ to construct a linear gradient in the wavelength domain. (c) Zoom-in picture for the $426 H N A$ array at four selected wavelengths (i. $4.67 \mu \mathrm{m}$, ii. $5.66 \mu \mathrm{m}$, iii. $6.88 \mu \mathrm{m}$, iv. $7.46 \mu \mathrm{m}$ ). The 427 expected pixel is illuminated at a resonant wavelength while other pixels are dark. The pixel is 428 illuminated at HNA resonance, which are $4.67 \mu \mathrm{m}$ for P1, $5.66 \mu \mathrm{m}$ for P6, $6.88 \mu \mathrm{m}$ for P12, and $4297.46 \mu \mathrm{m}$ for P16. (d) The hyperspectral image of HNA array at 16 resonance wavelengths for 16 430 pixels under different liquid analytes conditions (Acetone, IPA and Acetone: IPA=1:1). (e) The 431 extracted normalized reflection and difference normalized reflection spectrum of P1 to P16 under 432 molecules of acetone (I, IV), IPA (II, V), and Acetone: IPA=1:1 (III, VI). The analytes are in the 433 liquid phase and signals are captured by the microfluidic integrated HNA array. At the absorption 434 wavelength of IPA ( $\lambda_{7-13}$ and $\left.\lambda_{16}\right)$ and acetone ( $\lambda_{3}, \lambda_{7-11}$ and $\left.\lambda_{14,15}\right)$, the HNA reflection drops at the 435 desired pixel. (f) The reconstructed fingerprint barcode image by integrating spectrum at working 436 wavelength of each pixel for acetone (I), IPA (II), and Acetone: IPA=1:1 (III). The darker of the 437 pixels refers to the stronger absorption of molecules. The absorption peaks of IPA at 7.0 to $8.0 \mu \mathrm{m}$ 438 and $8.5 \mu \mathrm{m}$ are captured by $P 9$ to $P 13$ and $P 16$ respectively, while the absorption peaks of acetone 439 at $5.74 \mu \mathrm{m}, 7.0$ to $7.5 \mu \mathrm{m}$ and $8.0 \mu \mathrm{m}$ are captured by $P 1$ to $P 3, P 9$ to $P 11$ and P14 respectively.

440 In the mixture sample, both absorption peaks are captures from the reconstructed imaging.

441

442

\section{References}

443 1. Zhu, J., Ren, Z. \& Lee, C. Toward Healthcare Diagnoses by Machine-Learning-Enabled Volatile Organic Compound Identification. ACS Nano 15, 894-903 (2021).

445 2. Hermans, J. J. et al. 2D-IR spectroscopy for oil paint conservation: Elucidating the water-sensitive structure of zinc carboxylate clusters in ionomers. Sci. Adv. 5, eaaw3592 (2019).

447 3. Shi, L. et al. Mid-infrared metabolic imaging with vibrational probes. Nat. Methods 17, 844-851 (2020).

448 4. Hu, F., Shi, L. \& Min, W. Biological imaging of chemical bonds by stimulated Raman scattering 449 microscopy. Nat. Methods 16, 830-842 (2019).

450 5. Colom, A. et al. A fluorescent membrane tension probe. Nat. Chem. 10, 1118-1125 (2018).

451 6. Naumov, A. V. et al. Micro-Refractometry and Local-Field Mapping with Single Molecules. Nano Lett. 18, $452 \quad 6129-6134(2018)$.

453 7. Hartstein, A., Kirtley, J. R. \& Tsang, J. C. Enhancement of the Infrared Absorption from Molecular Monolayers with Thin Metal Overlayers. Phys. Rev. Lett. 45, 201-204 (1980).

455 8. Neubrech, F. et al. Resonant Plasmonic and Vibrational Coupling in a Tailored Nanoantenna for Infrared Detection. Phys. Rev. Lett. 101, 157403 (2008).

4579 9. Adato, R. et al. Ultra-sensitive vibrational spectroscopy of protein monolayers with plasmonic nanoantenna arrays. Proc. Natl. Acad. Sci. 106, 19227-19232 (2009). 
10. Adato, R. \& Altug, H. In-situ ultra-sensitive infrared absorption spectroscopy of biomolecule interactions in real time with plasmonic nanoantennas. Nat. Commun. 4, 2154 (2013).

11. Adato, R., Artar, A., Erramilli, S. \& Altug, H. Engineered absorption enhancement and induced transparency in coupled molecular and plasmonic resonator systems. Nano Lett. 13, 2584-91 (2013).

12. Neubrech, F., Huck, C., Weber, K., Pucci, A. \& Giessen, H. Surface-Enhanced Infrared Spectroscopy Using Resonant Nanoantennas. Chem. Rev. 117, 5110-5145 (2017).

13. Ren, Z. et al. Leveraging of MEMS Technologies for Optical Metamaterials Applications. Adv. Opt. Mater. 8, $1900653(2020)$.

14. Gao, B. et al. Nanoscale Refractive Index Sensors with High Figures of Merit via Optical Slot Antennas. ACS Nano 13, 9131-9138 (2019).

15. Zhan, C., Liu, B.-W., Tian, Z.-Q. \& Ren, B. Determining the Interfacial Refractive Index via Ultrasensitive Plasmonic Sensors. J. Am. Chem. Soc. 142, 10905-10909 (2020).

16. Semenyshyn, R. et al. In Vitro Monitoring Conformational Changes of Polypeptide Monolayers Using Infrared Plasmonic Nanoantennas. Nano Lett. 19, 1-7 (2019).

17. Kühner, L. et al. Vibrational Sensing Using Infrared Nanoantennas: Toward the Noninvasive Quantitation of Physiological Levels of Glucose and Fructose. ACS Sensors 4, 1973-1979 (2019).

18. Limaj, O. et al. Infrared Plasmonic Biosensor for Real-Time and Label-Free Monitoring of Lipid Membranes. Nano Lett. 16, 1502-1508 (2016).

19. Etezadi, D. et al. Nanoplasmonic mid-infrared biosensor for in vitro protein secondary structure detection. Light Sci. Appl. 6, e17029-e17029 (2017).

20. Hasan, D. \& Lee, C. Hybrid Metamaterial Absorber Platform for Sensing of CO2 Gas at Mid-IR. Adv. Sci. 5, (2018).

21. Hu, H. et al. Gas identification with graphene plasmons. Nat. Commun. 10, 1131 (2019).

22. Zhou, H. et al. Metal-Organic Framework-Surface-Enhanced Infrared Absorption Platform Enables Simultaneous On-Chip Sensing of Greenhouse Gases. Adv. Sci. 7, 2001173 (2020).

23. Yoo, D. et al. Low-Power Optical Trapping of Nanoparticles and Proteins with Resonant Coaxial Nanoaperture Using 10 nm Gap. Nano Lett. 18, 3637-3642 (2018).

24. Miao, X., Yan, L., Wu, Y. \& Liu, P. Q. High-sensitivity nanophotonic sensors with passive trapping of 
analyte molecules in hot spots. Light Sci. Appl. 10, 5 (2021).

25. Huck, C. et al. Surface-Enhanced Infrared Spectroscopy Using Nanometer-Sized Gaps. ACS Nano 8, 49084914 (2014).

490 26. Dong, L. et al. Nanogapped Au Antennas for Ultrasensitive Surface-Enhanced Infrared Absorption Spectroscopy. Nano Lett. 17, 5768-5774 (2017).

492 27. Su, D.-S., Tsai, D. P., Yen, T.-J. \& Tanaka, T. Ultrasensitive and Selective Gas Sensor Based on a Channel Plasmonic Structure with an Enormous Hot Spot Region. ACS Sensors 4, 2900-2907 (2019).

28. Zhu, Y. et al. Optical conductivity-based ultrasensitive mid-infrared biosensing on a hybrid metasurface. Light Sci. Appl. 7, 67 (2018).

29. Lee, I.-H., Yoo, D., Avouris, P., Low, T. \& Oh, S.-H. Graphene acoustic plasmon resonator for ultrasensitive infrared spectroscopy. Nat. Nanotechnol. 14, 313-319 (2019).

30. Garcia de Abajo, F. J. et al. Mid-infrared plasmonic biosensing with graphene. Science (80-. ). 349, 165-168 (2015).

31. Le, T. H. H. \& Tanaka, T. Plasmonics-Nanofluidics Hydrid Metamaterial: An Ultrasensitive Platform for Infrared Absorption Spectroscopy and Quantitative Measurement of Molecules. ACS Nano 11, 9780-9788

32. Xu, J. et al. Nanometer-Scale Heterogeneous Interfacial Sapphire Wafer Bonding for Enabling PlasmonicEnhanced Nanofluidic Mid-Infrared Spectroscopy. ACS Nano 14, 12159-12172 (2020).

505 33. Wei, J. et al. Ultrasensitive Transmissive Infrared Spectroscopy via Loss Engineering of Metallic 506 Nanoantennas for Compact Devices. ACS Appl. Mater. Interfaces 11, 47270-47278 (2019).

507 34. Rodrigo, D. et al. Resolving molecule-specific information in dynamic lipid membrane processes with 508 multi-resonant infrared metasurfaces. Nat. Commun. 9, (2018).

509 35. Hasan, D., Ho, C. P. \& Lee, C. Realization of Fractal-Inspired Thermoresponsive Quasi-3D Plasmonic Metasurfaces with EOT-Like Transmission for Volumetric and Multispectral Detection in the Mid-IR Region. ACS Omega 1, 818-831 (2016).

512 36. Rodrigo, D., Tittl, A., John-Herpin, A., Limaj, O. \& Altug, H. Self-Similar Multiresonant Nanoantenna 513 Arrays for Sensing from Near- to Mid-Infrared. ACS Photonics 5, 4903-4911 (2018).

514 37. Tittl, A. et al. Imaging-based molecular barcoding with pixelated dielectric metasurfaces. Science (80-. ). 
360, 1105-1109 (2018).

516 38. Leitis, A. et al. Angle-multiplexed all-dielectric metasurfaces for broadband molecular fingerprint retrieval.

517 Sci. Adv. 5, eaaw2871 (2019).

518 39. Kühner, L. et al. Nanoantenna-Enhanced Infrared Spectroscopic Chemical Imaging. ACS Sensors 2, 655-

$519 \quad 662(2017)$.

520 40. Yesilkoy, F. et al. Ultrasensitive hyperspectral imaging and biodetection enabled by dielectric metasurfaces. Nat. Photonics 13, 390-396 (2019).

522

523 Methods

\section{TCMT modeling:}

525 The temporal coupled-mode theory (TCMT) is used to model the coupling behavior between PNA

526 and molecular vibration. We treat the plasmonic resonant (denoted as P) as a bright mode that is 527 coupled to the incident light, while we treat the molecular vibration (denoted as M) as a dark mode, 528 which coupling efficiency is much lower than PNA and can be ignored in their coupling system. 529 Therefore, we write down the equations using TCMT as

$$
\frac{d P}{d t}=j \omega_{0} P-\left(\gamma_{a}+\gamma_{r}\right) P+j \mu M+\sqrt{\gamma_{r}} S_{i n}
$$

$$
\frac{d M}{d t}=j \omega_{m} M-\gamma_{m} M+j \mu P
$$

$$
S_{t}=S_{\text {in }}-\sqrt{\gamma_{r}} P
$$

$$
S_{r}=\sqrt{\gamma_{r}} P
$$

534 where $P, M$ and $\omega_{0,} \omega_{m}$ represent the amplitude and angular frequency of resonance for HNA and 535 molecular vibration, respectively. $\gamma_{a}$ and $\gamma_{r}$ denote the radiative and absorptive losses of HNA, while $\gamma_{m}$ is the absorptive loss of molecules. $\mu$ is the coupling strength between HNA and molecular

537 vibration. $S_{i n}$, $S_{t}$, and $S_{r}$ represent the amplitude of incident, transmitted, and reflected light, 
538 respectively. Under the time-harmonic condition, the first derivative of time $(d / d t)$ is replaced by $539 j \omega$, and the Eq.1 and Eq.2 can be simplified as

$$
\left[j\left(\omega-\omega_{0}\right)+\left(\gamma_{a}+\gamma_{r}\right)\right] P=j \mu M+\sqrt{\gamma_{r}} S_{i n}
$$

$$
\left[j\left(\omega-\omega_{0}\right)+\gamma_{m}\right] M=j \mu P
$$

By substituting eq. 6 to eq.5, we can eliminate $M$ and obtain

$$
\left[j\left(\omega-\omega_{0}\right)+\left(\gamma_{a}+\gamma_{r}\right)+\frac{\mu^{2}}{j\left(\omega-\omega_{0}\right)+\gamma_{m}}\right] P=\sqrt{\gamma_{r}} S_{\text {in }}
$$

544 Therefore, $\mathrm{T}$ and $\mathrm{R}$ can be expressed as

$$
T(\omega), R(\omega)=\left|\frac{S_{\mathrm{t}, \mathrm{r}}}{S_{\mathrm{in}}}\right|^{2}=\left|\frac{j\left(\omega-\omega_{0}\right)+\gamma_{a, r}+\frac{\mu^{2}}{j\left(\omega-\omega_{m}\right)+\gamma_{m}}}{j\left(\omega-\omega_{0}\right)+\left(\gamma_{a}+\gamma_{r}\right)+\frac{\mu^{2}}{j\left(\omega-\omega_{m}\right)+\gamma_{m}}}\right|^{2}
$$

546 The EIT-like line shape and Fano-like line shape can be expressed from Eq.8 when $\omega_{0}=\omega_{m}$ and $547 \omega_{0} \neq \omega_{m}$, respectively. The plasmonic resonance can be easily obtained when there is no coupling 548 effect from molecules $(\mu=0)$.

$$
T(\omega)=\frac{\left(\omega-\omega_{0}\right)^{2}+\gamma_{r}^{2}}{\left(\omega-\omega_{0}\right)^{2}+\left(\gamma_{a}+\gamma_{r}\right)^{2}}
$$

$$
R(\omega)=\frac{\gamma_{a}{ }^{2}}{\left(\omega-\omega_{0}\right)^{2}+\left(\gamma_{a}+\gamma_{r}\right)^{2}}
$$

$$
A(\omega)=1-T-R=\frac{2 \gamma_{a} \gamma_{r}}{\left(\omega-\omega_{0}\right)^{2}+\left(\gamma_{a}+\gamma_{r}\right)^{2}}
$$

552 Eq. 9-11 is used to extract absorptive and radiative loss of HNA by fitting the resonance spectrum 553 in the frequency domain from simulation (Extended Data Fig.1). By engineering the HNA 
554 structure by changing $\Delta \mathrm{L}$ with the constant $\mathrm{L}$, the $\gamma_{r}$ and $\gamma_{a}$ can be tuned continuously, and $\omega_{0}$

555 remains unchanged. To explore the sensing performance, we have made some assumptions to

556 simplify Eq.8 in order to perform the analytical operation. First, we make $\omega_{0}=\omega_{m}$ to match the

557 frequency of HNA and molecular vibration since the WGHNA is only designed for the molecular

558 absorption wavelength near the HNA resonance wavelength to have the best enhancement. Second,

559 we treat $\mu$ as a much smaller parameter compared with $\gamma_{m}, \gamma_{r}$, and $\gamma_{a}$. Therefore, we apply the 560 difference between Eq.9 and Eq.8 when $\omega=\omega_{0}=\omega_{m}$.

561

$$
\Delta T\left(\omega=\omega_{0}\right)=\frac{2 \mu^{2}}{\gamma_{m}} \frac{\gamma_{a} \gamma_{r}}{\left(\gamma_{a}+\gamma_{r}\right)^{2}\left(\gamma_{a}+\gamma_{r}+\frac{\mu^{2}}{\gamma_{m}}\right)}+\left(\frac{\mu^{2}}{\gamma_{m}}\right)^{2} \frac{\gamma_{r}^{2}}{\left(\gamma_{a}+\gamma_{r}\right)^{2}\left(\gamma_{a}+\gamma_{r}+\frac{\mu^{2}}{\gamma_{m}}\right)^{2}}
$$

562 Since $\mu<<\gamma_{m}, \frac{\mu^{2}}{\gamma_{m}}$ is a small real number close to 0 . Therefore, we cancel the high order term and 563 simplify Eq.12 as

$$
\Delta T\left(\omega=\omega_{0}\right)=\frac{2 \mu^{2}}{\gamma_{a} \gamma_{m}} \frac{f}{(1+f)^{3}}
$$

$$
\Delta R\left(\omega=\omega_{0}\right)=-\frac{2 \mu^{2}}{\gamma_{a} \gamma_{m}} \frac{f^{2}}{(1+f)^{3}}
$$

568 The negative sign in Eq.14 indicates the opposite change in transmission and reflection spectrum 569 induced by molecules vibration. $\gamma_{a}$ refers to the omics loss of material; thus, it is constant in our 570 experiment of HNA made by $\mathrm{Au}$. When changing $\Delta \mathrm{L}$ of HNA, the electrical field does not change 571 too much among different HNA devices, so that $\mu$ is also a constant. Additionally, $\gamma_{\mathrm{m}}$ is also 572 unchanged since we fix the absorption peaks of the " $\mathrm{C}=\mathrm{O}$ " bond from PMMA in sensitivity 
573 characterization. By applying the first derivative of $f$ for Eq.13 and Eq.14. We further calculate

574 the maximum enhancement of the $\mathrm{T}$ and $\mathrm{R}$ spectrum and get the optimal condition that occurs

575 when $\mathrm{f}$ equals 0.5 and 2 , respectively.

576 FDTD simulation:

577 The finite-difference time-domain (FDTD) method (Lumerical FDTD ${ }^{1}$ ) is performed to simulate 578 the far-field spectrum and the nearfield distribution of plasmonic hook nanoantenna. The light 579 source is selected as a plane-wave to simulate the incidence of light from free space. The incidence 580 angle and polarization state are adjusted to the desired orientation to excite the bipolar mode 581 plasmonic resonance of HNA. The refractive index of $\mathrm{CaF}_{2}$ is set at 1.38 at wavelengths ranging 582 from $2 \mu \mathrm{m}$ to $10 \mu \mathrm{m}$. The periodic boundary at the $\mathrm{x}$ and y-axis (Fig. 2 a) is selected to simulate 583 the effect of the periodic antenna array, and the PML boundary is chosen at the z-axis to transport 584 light into free space. The electric field and magnetic field of HNA with different $\Delta \mathrm{L}$ are plotted in 585 Extended Data Fig.2. The electrical field distribution reflects the electric field's intensity and 586 polarity at the resonance wavelength of nanorod and HNA devices when $\Delta \mathrm{L}$ changes. The $\mathrm{E}^{2}$ 587 intensity remains the same at all devices representing the constant coupling efficiency $\mu$. 588 Additionally, Ex polarity indicates the bipolar resonance mode at resonance wavelength of all 589 nanorod and HNA devices when $\Delta \mathrm{L}$ changes. Furthermore, magnetic field distribution reflects the 590 current orientation at resonance wavelength, indicating the nanoantenna's radiation capability. As $591 \Delta \mathrm{L}$ increase, the overlap of inverse current at the short arm of HNA ruin the radiation due to the 592 large radiative loss $\left(\gamma_{r}\right)$. Therefore, the HNA become dark as the transmission and reflection drops. 
595 For the fabrication of HNA, electron-beam lithography (EBL, Jeol 6500FS) and lift-off process is

596 used to pattern the nanometer scale gold structure. Before EBL, the $\mathrm{CaF}_{2}$ chip was firstly rinsed

597 by Acetone and IPA solutions for 1 min with sonication. After that, the chip is treated under oxygen

598 plasma for the uniform formation of PMMA 495K A5 photoresist, which is spin-coated at 4000

$599 \mathrm{rpm}$ for $1 \mathrm{~min}$. Since the conductivity of the $\mathrm{CaF}_{2}$ chip is low, an additional E-spacer layer is spin-

600 coated at $2000 \mathrm{rpm}$ for $1 \mathrm{~min}$ to avoid charge accumulation during EBL. After EBL, the

601 development with $30 \mathrm{~s}$ using PMMA developer (MIBK:IPA=1:3) is used to remove PMMA resist

602 under exposure following by the cleaning with IPA for $30 \mathrm{~s}$. Then electron beam evaporation (AJA

603 International Inc.) is proceeded to deposit $80 \mathrm{~nm}$ thick gold on top of $\mathrm{CaF}_{2}$ substrate and PMMA

604 photoresist. To lift-off the nanoantenna pattern, the chip is placed in acetone for one day and rinsed

605 by IPA. After that, the HNA chip is tested by SEM (Hitachi) and AFM (Bruker FastScan) to

606 confirm the geometry (Extended Data Fig.3).

607

608 FTIR measurement:

609 A Fourier-transformed IR (FTIR) microscope (Agilent Cary 660) with an FTIR spectrometer 610 (Agilent Cary 620) and $\mathrm{HgCdTe}$ (mercury cadmium telluride, MCT) detector is used to

611 characterize hook nanoantenna. The background signal is collected from the $\mathrm{CaF}_{2}$ chip using 16-

61232 scans at $8 \mathrm{~cm}^{-1}$ resolution to compensate for the MIR gas absorption (mainly water vapor and $613 \mathrm{CO}_{2}$ ) from the ambient. Then the sample scan is performed using $16-32$ scans at $8 \mathrm{~cm}^{-1}$ resolution

614 to capture the spectral response of nanoantenna. The scanning area is adjusted to $200 * 200 \mu \mathrm{m}^{2}$ to

615 fit the nanoantenna area. For liquid sensing, a microfluidic chamber made by PDMS is bonded to

616 a $\mathrm{CaF}_{2}$ chip to allow the contact of the liquid analyte with HNA, and the spectrum is captured 617 simultaneously. 
618 The hyperspectral images are captured by an IR focal plane array (FPA) MCT detector with $32 * 32$

619 pixels. To get the IR spectrum of each pixels of the HNA array, the alignment of HNA position is 620 performed at each data acquisitions. Due to the mutual coupling of HNA array, we cannot fit one 621 HNA pixel to one FPA pixel to get a perfect image. Therefore, we need to fit one HNA pixel to 622 multiple FPA pixels as shown in Extended Data Fig.4. We fix the pixel number of HNA array 623 and change the pixel area. For pixel area of $40 * 40 \mu \mathrm{m}^{2}$, we achieve the data acquisitions in one 624 time (Extended Data Fig.4 b). For pixel area of $80 * 80 \mu \mathrm{m}^{2}$, we achieve the data acquisitions in 625 four times (Extended Data Fig.4 c). Compared with these two figures, a clearer square-shape 626 imaging of HNA array is shown in the picture by 4 pixels per acquisition. This result shows that 627 the spatial resolution of HNA array is mainly limited by pixels of FPA, which can be improved by 628 replacing the FPA with more pixel numbers, smaller pixel area, and better detectivity (D*).

629

\section{Visualization using PCA}

631 Principal component analysis is used in exploratory data analysis and for making predictive models.

632 It is commonly used for dimensionality reduction by projecting each data point onto only the first

633 few principal components to obtain lower-dimensional data while preserving as much of the data's

634 variation as possible. To facilitate visualization of the feature space, PCA was performed in 635 MATLAB_R2020a. In this case, a covariance matrix was computed using a factorization of 636 singular value decomposition (SVD) for the normalized set of features from which the 637 eigenvectors and eigenvalues were extracted. Each principal component was constructed as a 638 linear combination of the initial features. The first three principal components were then used to 639 display $3 \mathrm{D}$ scatter plots of the features. 
640 In our experiment, we select methanol, ethanol and IPA as a proof-of-concept demonstration and

641 dilute the liquid analytes into water with a constant volume concentration of $1 \%$. The IR fingerprint

642 absorption peaks of these analytes are shown in Extended Data Fig. 5 d. By mixing the two of

643 three analytes, we have six different analyte states, which are $1 \%$ methanol, $1 \%$ ethanol, $1 \%$ IPA,

$6441 \%$ methanol $+1 \%$ ethanol, and $1 \%$ methanol $+1 \%$ IPA. To compare the device performance, we

645 have four device configurations, which are HNA, HNA supercell, nanorod antenna (NA), NA

646 supercell. For each analyte states and device configurations, we measure 50 spectrums used for

647 ML. The identification results are shown in Fig. 5 e,f for HNA and HNA supercell and Extended

648 Fig.5 e,f for NA and NA supercell.
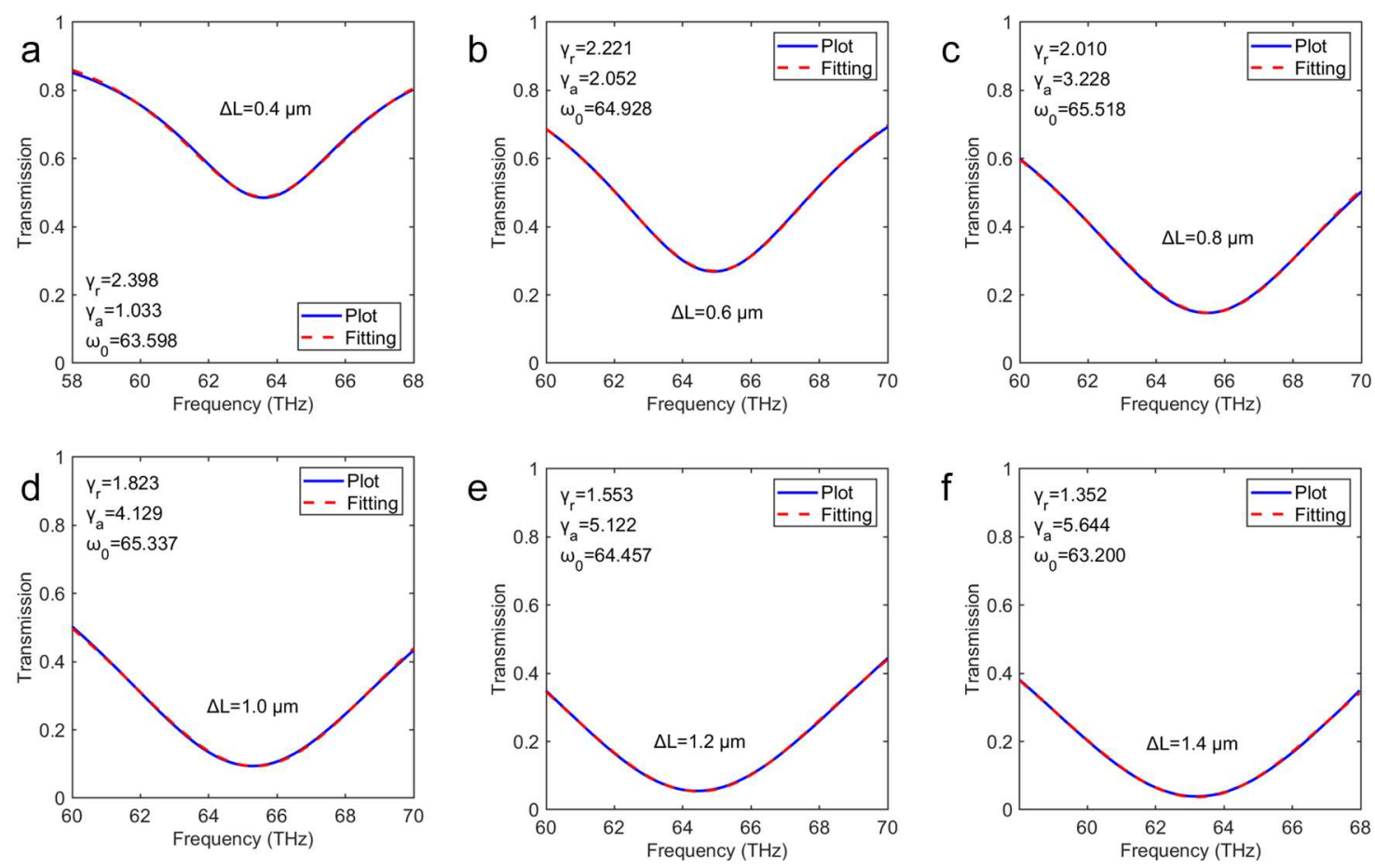

650 Extended Data Fig.1 Theoretical fitting of transmission spectrum of HNA at different $\triangle L$ using 

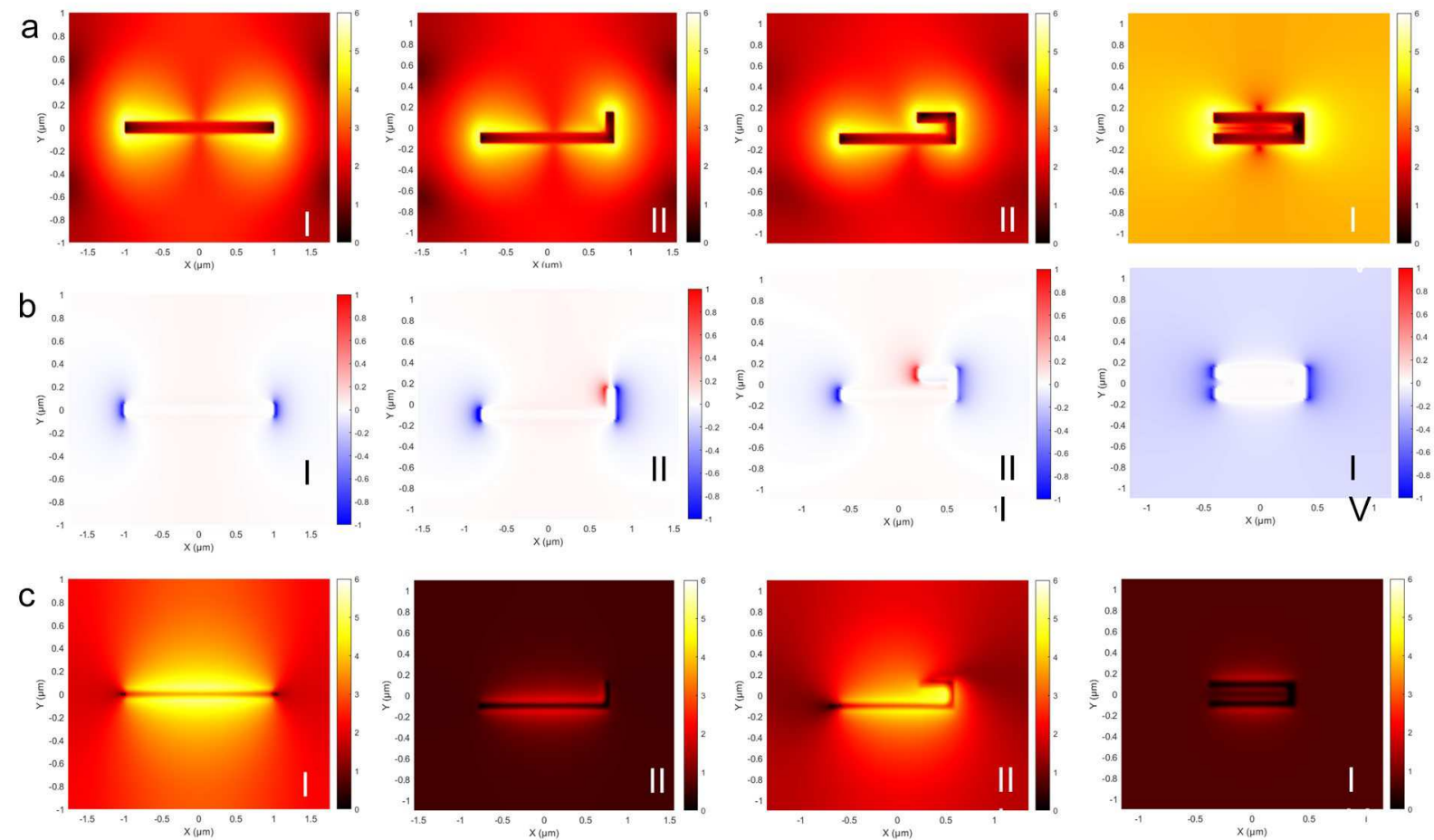

652
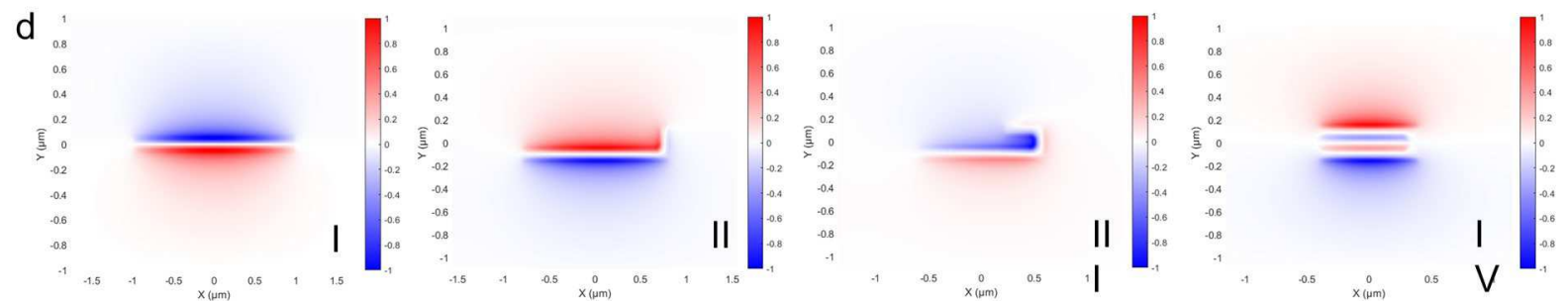

653 Extended Data Fig.2 FDTD simulation results of electric (a,b) and magnetic (c,d) field

654 distribution of nanoantenna by different folding degree.
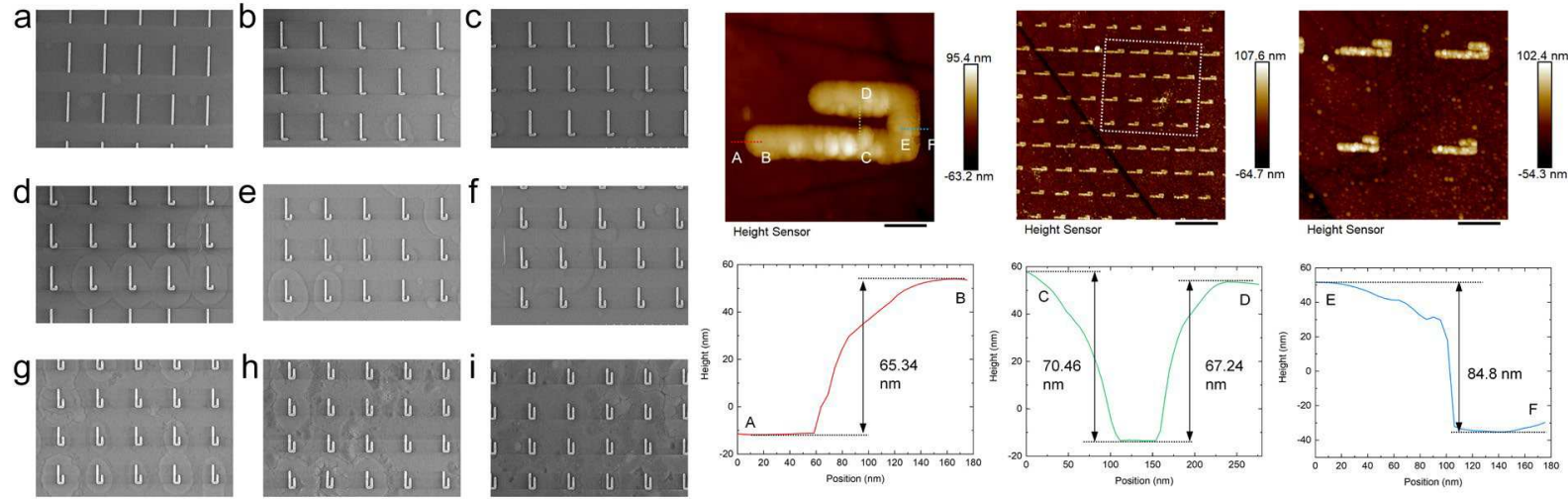

656 Extended Data Fig.3 SEM and AFM characterization of HNA. 


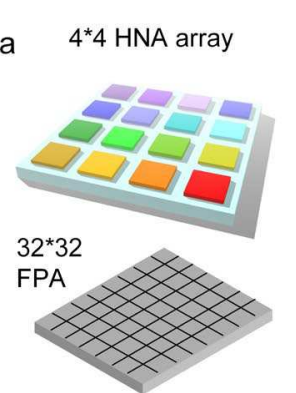

16 pixels per acquisition

b

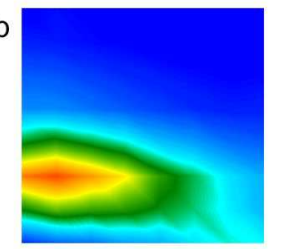

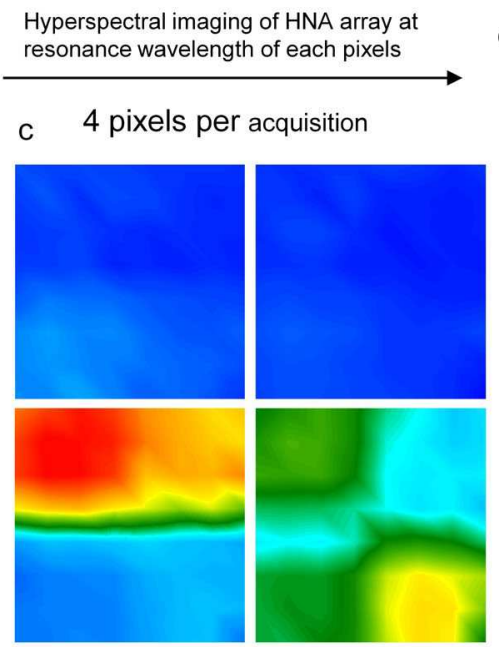

$\lambda=5.78 \mathrm{um}$
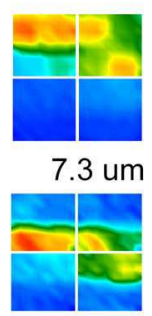

$6.61 \mathrm{um}$

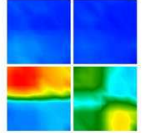

5.78 um

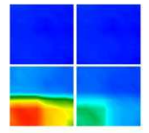

5.06 um

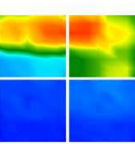

7.5 um

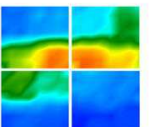

6.74 um

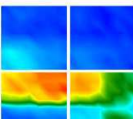

5.95 um

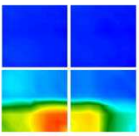

5.24 um

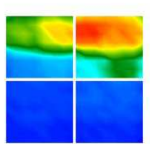

$7.62 \mathrm{um}$

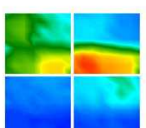

6.88 um

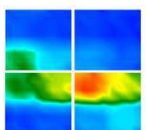

6.1 um

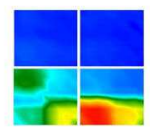

5.41 um

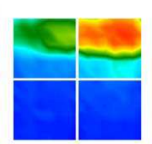

$7.8 \mathrm{um}$

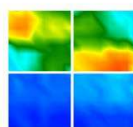

7.13 um

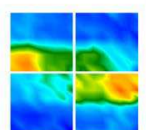

6.3 um

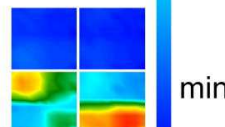

$5.58 \mathrm{um}$ $\max$

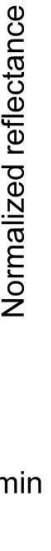

658 Extended Data Fig.4 (a) Schematic illustration of $4 * 4$ HNA array and $32 * 32$ FPA. (b) The

659 hyperspectral imaging of bare HNA array at $5.78 \mu \mathrm{m}$ for $H N A$ pixel area of $40^{*} 40 \mu m^{2}$ with one

660 data acquisition. (c) The hyperspectral imaging of bare HNA array at $5.78 \mu \mathrm{m}$ for HNA pixel area

661 of $80 * 80 \mu \mathrm{m}^{2}$ with four data acquisitions. (d) Hyperspectral imaging of HNA array at resonance wavelength of each pixels with four data acquisitions.
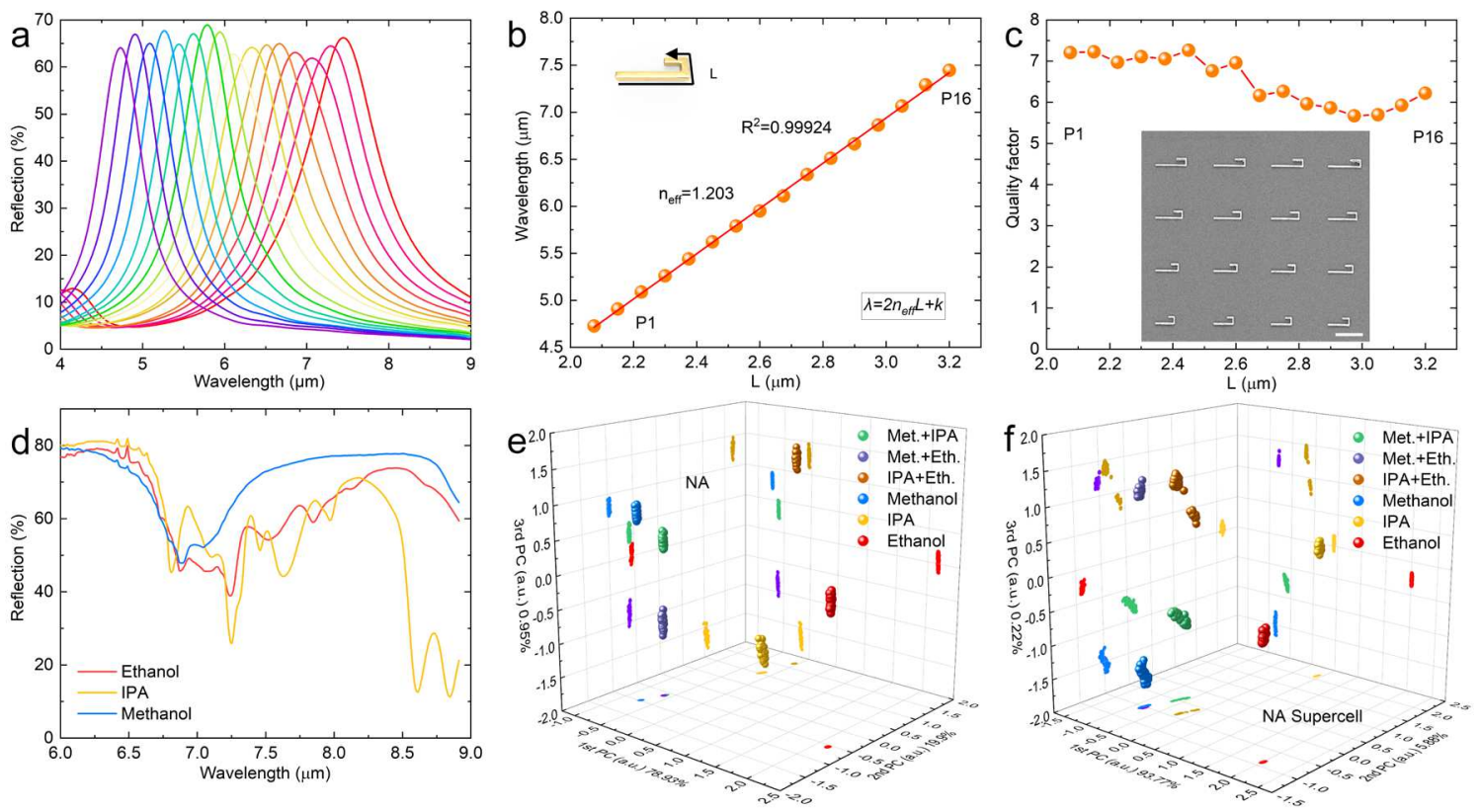
Extended Data Fig.5 (a) The reflection spectra of 16 HNA pixels measured by FTIR. (b)The linear

665 666 667 668 669 670 671

672

673

674 675 corresponding author on request.

676

677 Acknowledgements

678 This work was supported by the research grant of CRP-15th (NRF-CRP15-2015-02) and the 679 research grant of RIE 2020 (A18A5b0056) at the NUS, Singapore.

680 authors prepared the manuscript.

\section{Author contributions}

Z.R. and C.L. generated the design concept. Z.R. performed antenna design, fabrication, and FTIR testing. Z.Z. performed machine learning algorithm and data analysis. J.W. conducted the theoretical model and advised on fabrication and FTIR testing. C. L. supervised the project. All

\section{Competing interests}


688 The authors declare no competing financial interests.

689

690 Additional information

691 Supplementary information is available for this paper.

692

693 Competing interests

694 The authors declare no competing financial interests. 

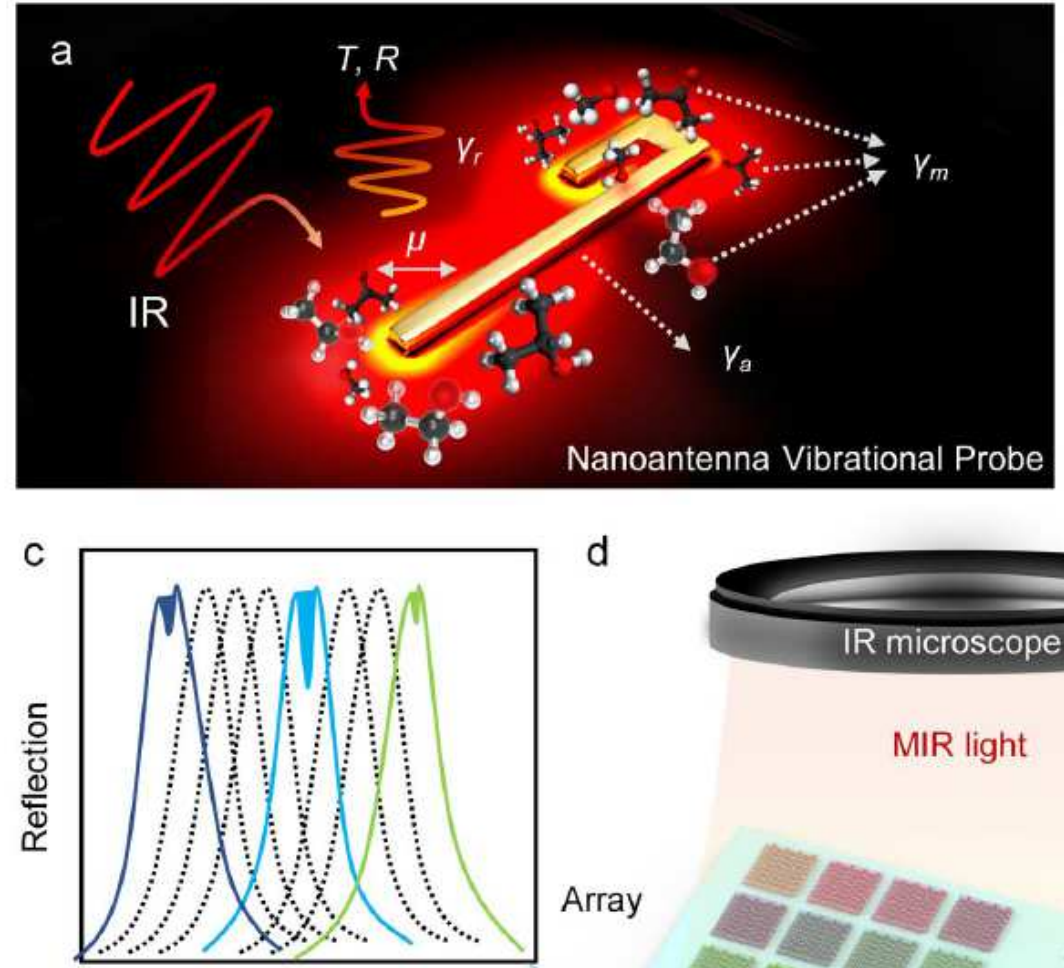

Wavelength

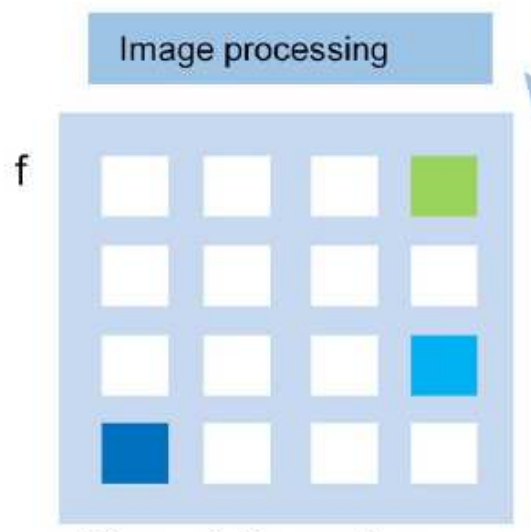

Fingerprint bar-coding
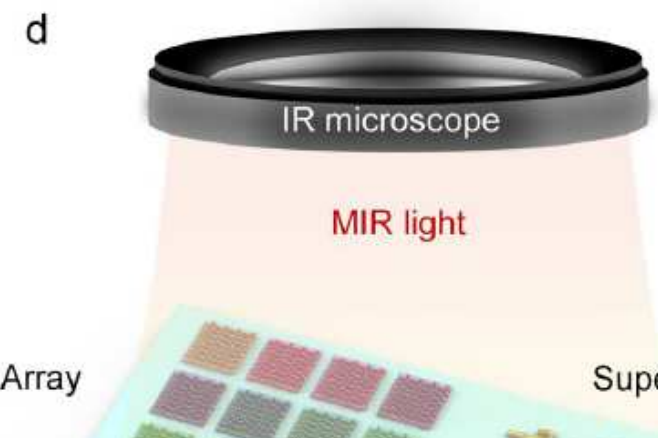

MIR light

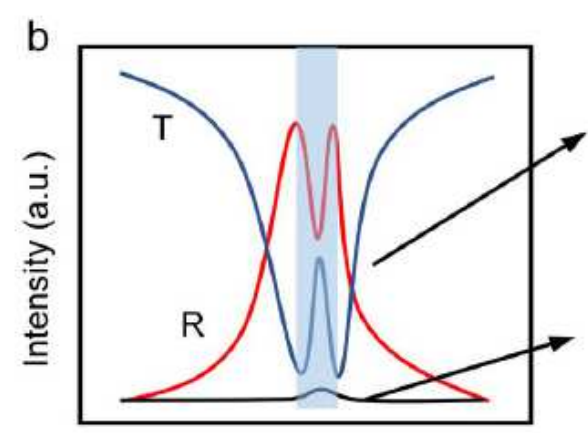

Wavelength
Enhanced molecular vibration

Intrinsic molecular vibration
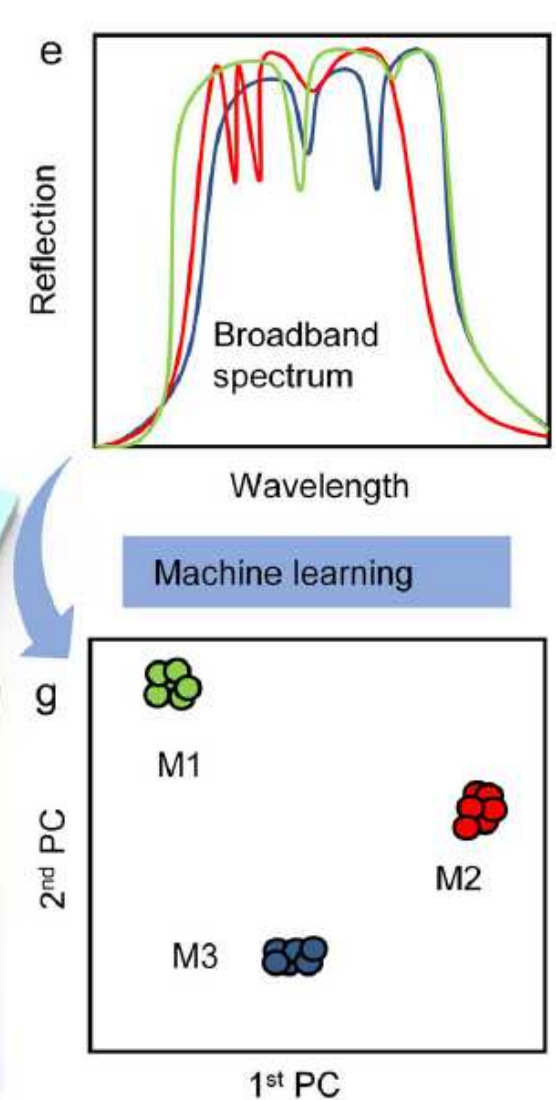

\section{Figure 1}

(a) Schematic drawing of HNA vibrational probe for molecular sensing by the interaction between plasmonic resonance and molecular vibration. The yellow color refers to the simulated electrical field near the surface of the hook antenna. The physical parameters ( $\gamma a, \gamma r, y m$ and $\mu$ ) are used in TCMT modeling to express the resonance behavior of PNA and molecular vibration. (b) The spectrum of plasmonic-enhanced molecular vibration signal in reflection $(R)$ and transmission $(T)$ compared with intrinsic fingerprint absorption. (c) The concept drawing of spectral response of wavelength-gradient HNA array with molecular vibration fingerprints. The different curves indicate the spectrum of each pixel in the HNA array. Each HNA pixel has one periodic HNA structure and the length of HNA gradually changes 
among different pixels. By gradually increasing the optical length of HNA in one pixel, the optical resonant wavelength is also increasing linearly. The molecular vibration is captured by the HNA pixel which operates at the same wavelength. The enhanced absorption is marked as shadow by different color for different pixels. (d) Schematic drawing of two types of WGHNA designs - HNA array and HNA supercell. Both HNA array and supercell have the wavelength gradient nanoantenna structure. The difference is that the wavelength gradient is designed at different order of structures, which are pixel and cell levels. The HNA array has periodic HNA structure at each pixel and changes among different pixels, while the HNA supercell holds the gradient nanoantenna into one unit-cell called supercell and repeat the supercell to form periodic structures. The inset SEM image is the top-view of HNA, and the scale bar indicates $1 \mu \mathrm{m}$. (e) The concept drawing of spectral response of wavelength-gradient HNA supercell with molecular vibration fingerprints. Example illustration of different molecular vibration fingerprints are marked in of blue, green, and red. The broadband response of supercell shows the capability to capture multiple absorption peaks. (f) The fingerprint barcoding image is processed from the HNA array pixel signal in (c). (g) Molecular recognition results from the broadband spectrum of HNA using principal component analysis (PCA) algorithm from machine learning. Each cluster indicates one type of molecule. 

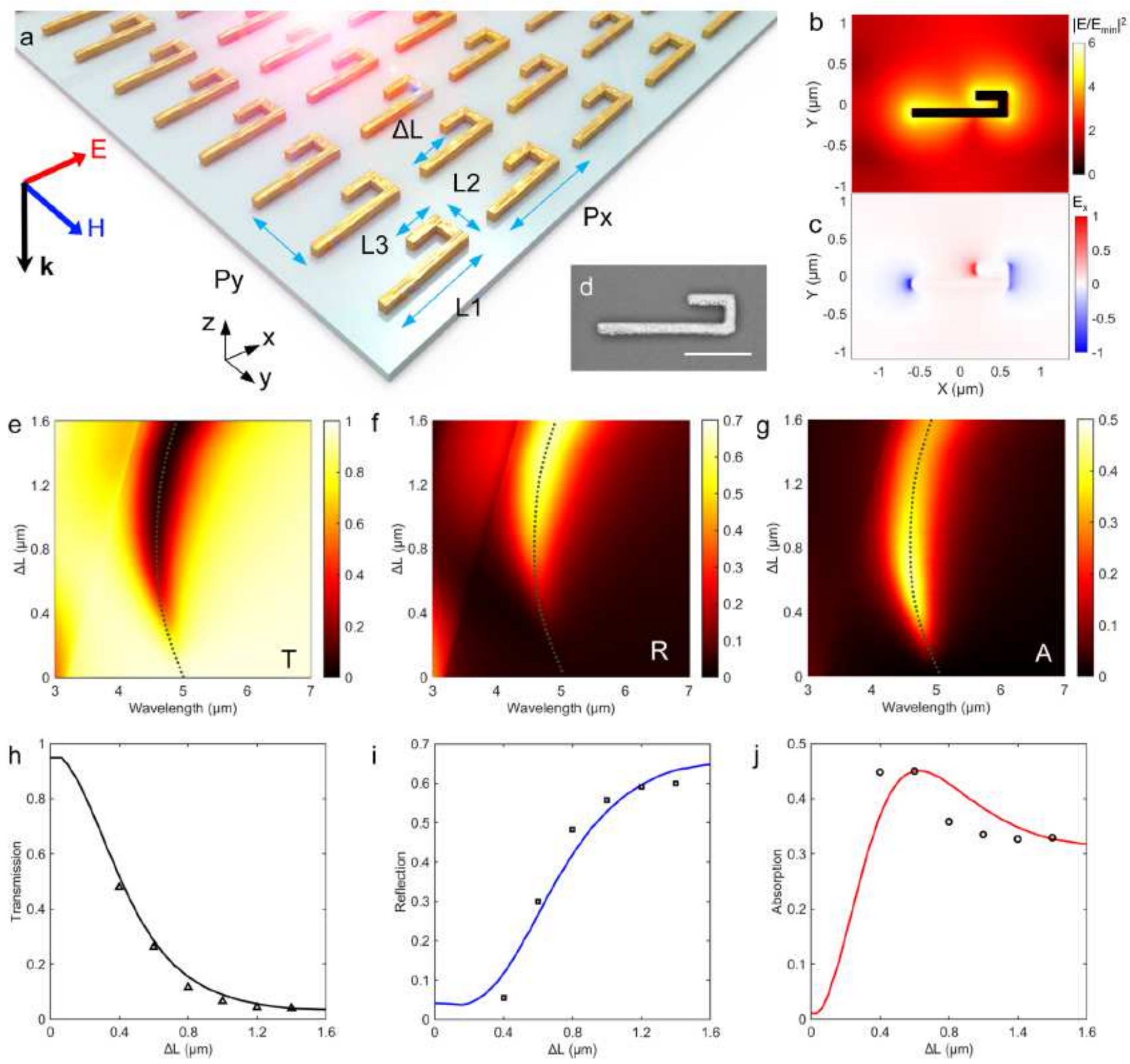

Figure 2

The plasmonic properties of HNA. (a) The schematic drawing of the HNA array. The polarization of incident light is aligned with the long arm of HNA. $\Delta \mathrm{L}$ is used to characterize the folding degree of the hook antenna. (b) Simulated nearfield distribution of electric field intensity of HNA when $\Delta \mathrm{L}$ equals 1.2 $\mu \mathrm{m}$. (c) Simulated nearfield distribution of electric field polarity of HNA when $\Delta \mathrm{L}$ equals $1.2 \mu \mathrm{m}$. The dipole resonance is generated at the resonance wavelength. (d)The SEM image of one HNA with scale bar indicating $500 \mathrm{~nm}$. (e-g) the IR response spectrum as the increment of $\Delta \mathrm{L}$ for transmission (e), reflection (f), and absorption (g). (h-i) The experimental results (points) as the increment of $\Delta \mathrm{L}$ compared with simulation results (curves). 

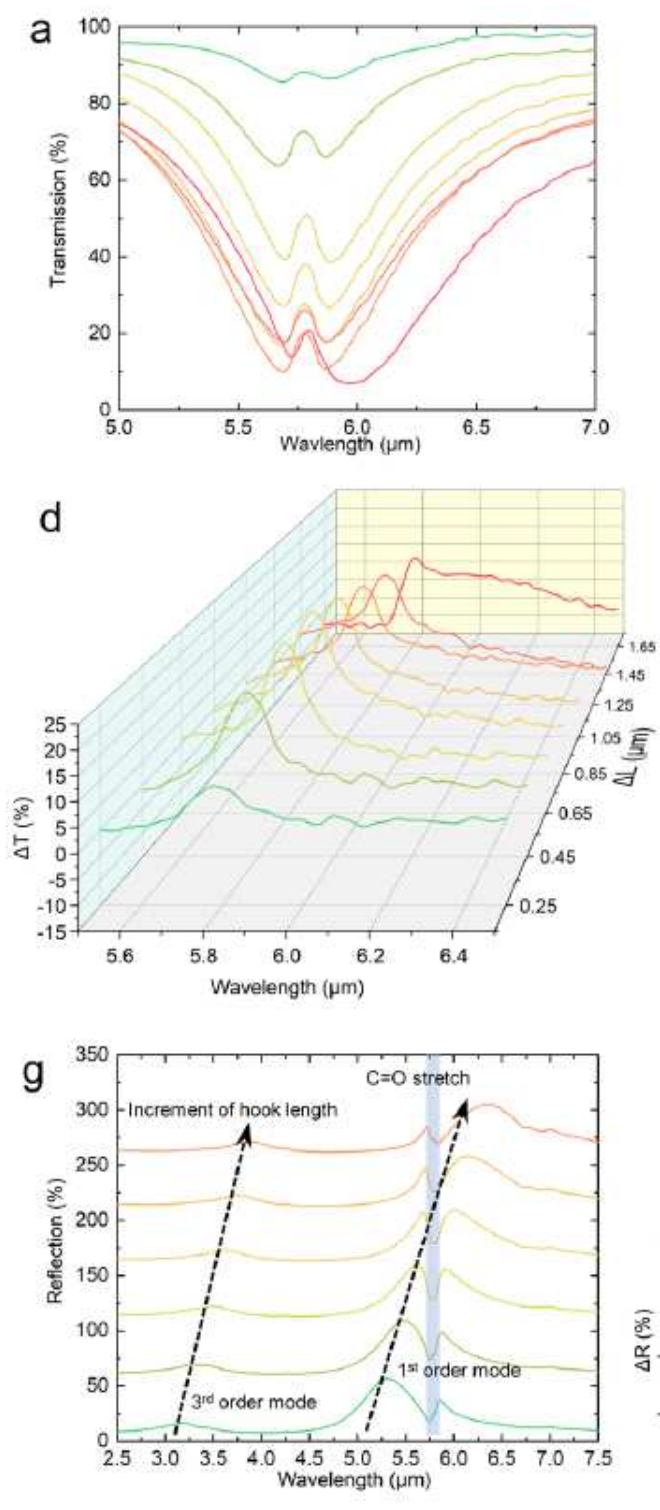
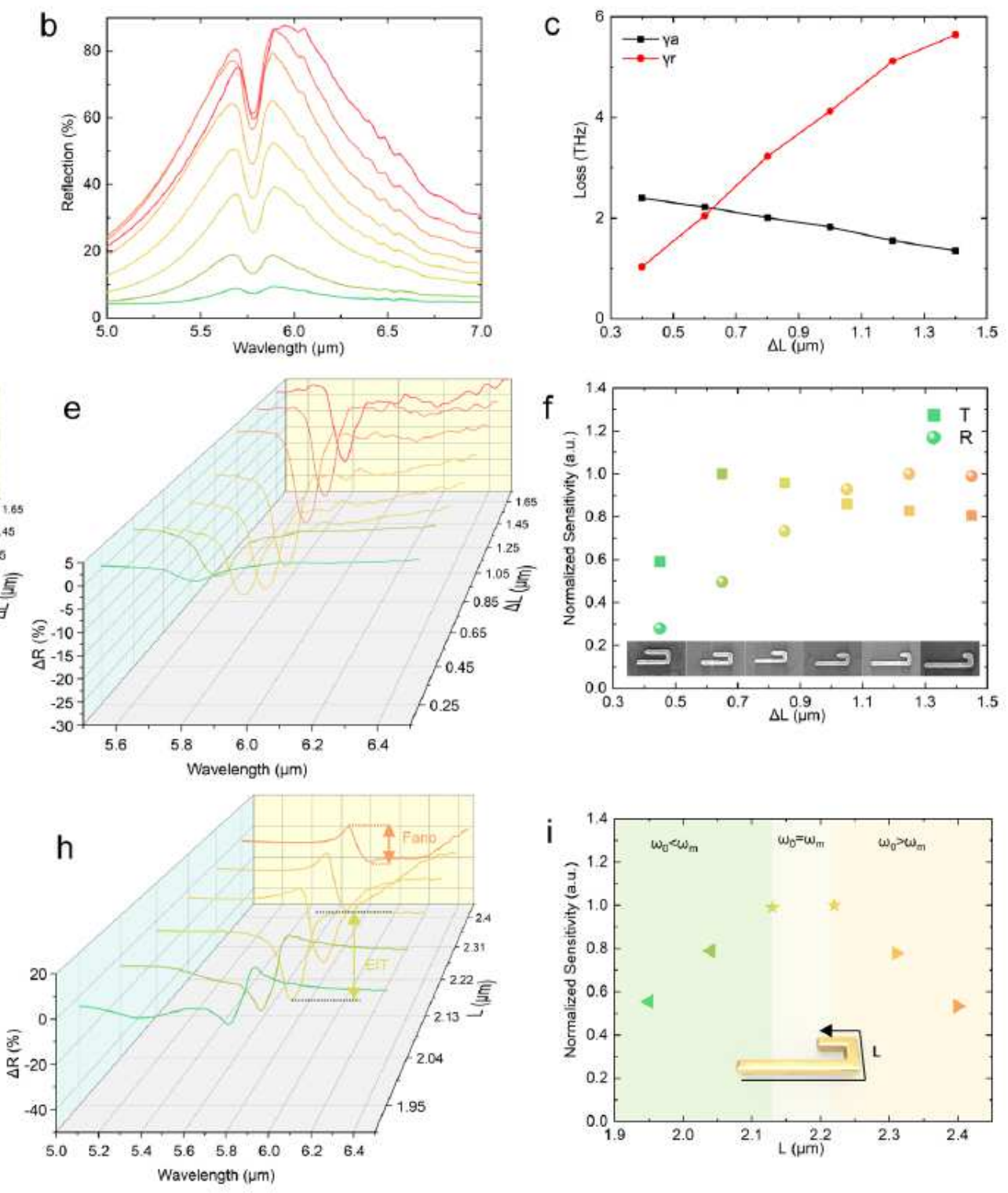

\section{Figure 3}

Characterization of sensing performance for HNA. $(a, b)$ Experimental sensing spectrum of HNA with variances of $\triangle \mathrm{L}$ for $\mathrm{C}=\mathrm{O}$ stretch of PMMA. (c)Theoretical fitting of damping rate of a different folded degree from TCMT. (d-e) Extracted sensing signal of HNA with a difference with reference devices. (e)the sensitivity of the HNA sensor of transmission and reflection mode with the change of $\Delta \mathrm{L}$. (g) experimental sensing spectrum of HNA with variances of $L$ for $C=0$ stretch of PMMA. (h) Extracted sensing signal of HNA with a difference with reference devices. (i) the sensitivity of the HNA sensor of transmission and reflection mode with a change of $L$. 

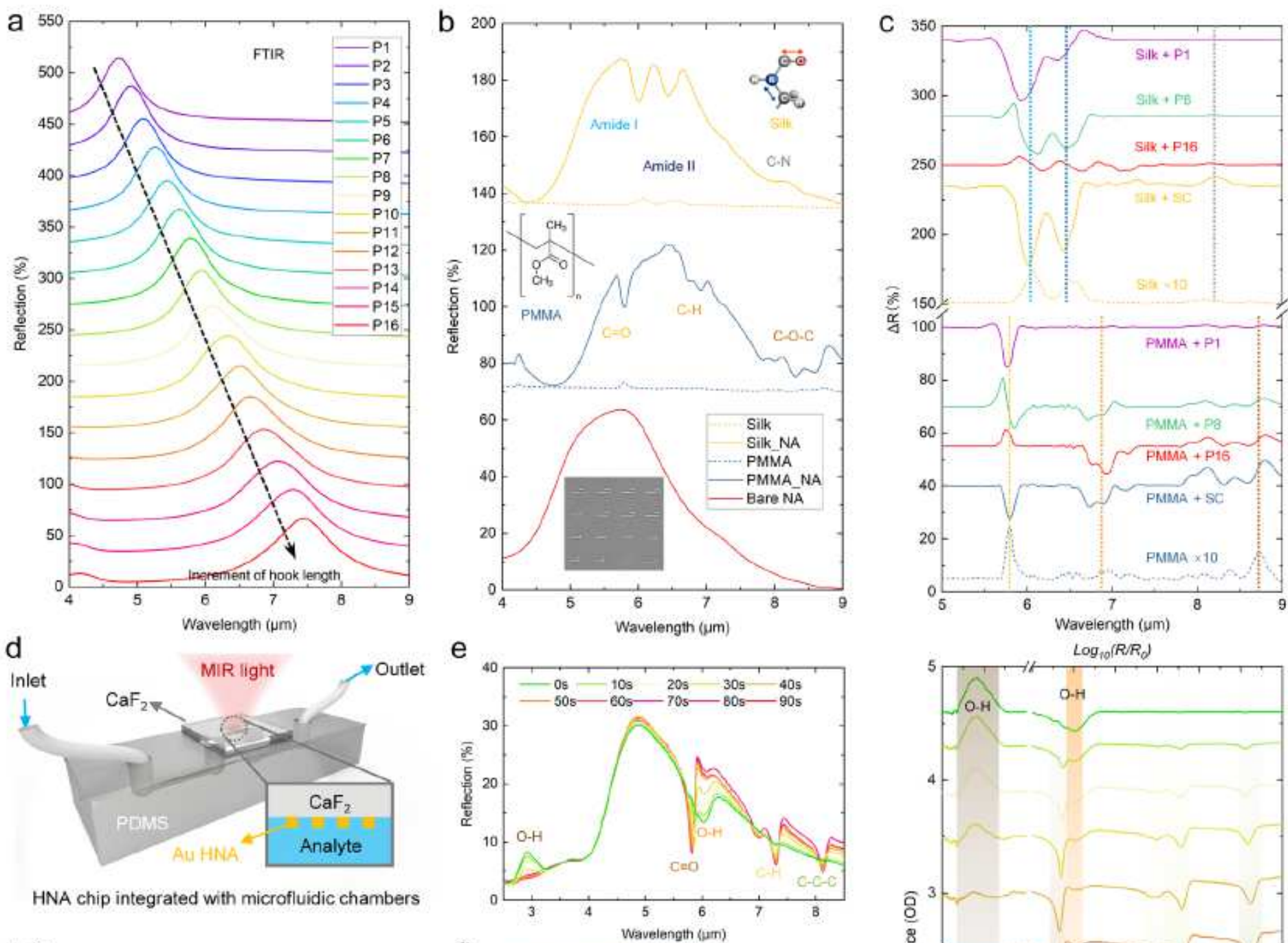

HNA chip integrated with microfluidic chambers
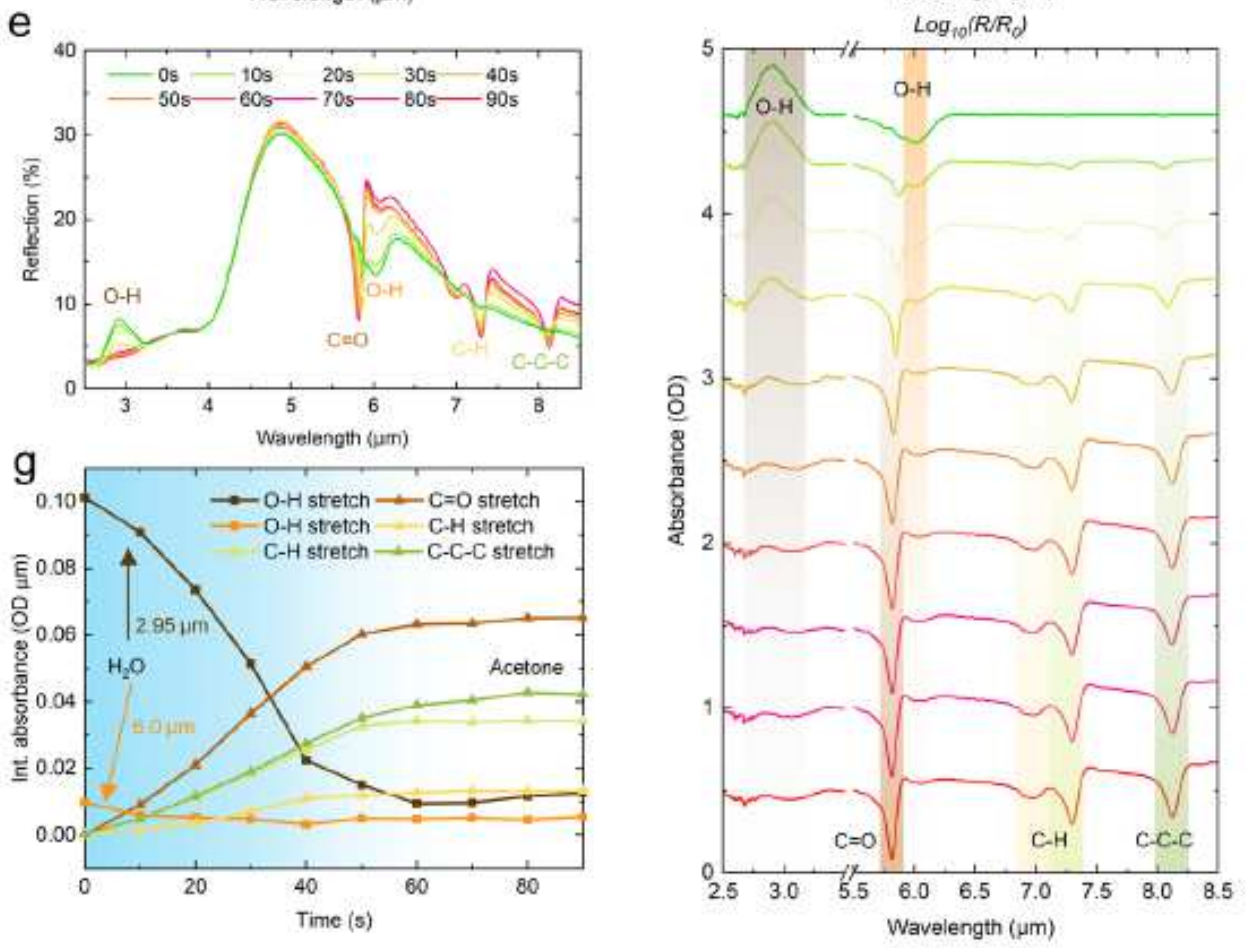

\section{Figure 4}

Broadband sensing characterization of HNA supercell by gradient increase HNA length into a unit cell and broadband monitoring of fluidic dynamics for acetone injection into the water from HNA supercell integrated with PDMS microfluidic chamber. (a) The experimental reflection spectrum of HNA by changing the length. A longer HNA leads to a longer resonant wavelength. By combing different lengths into a unit cell of the metasurface, and HNA supercell is formed with broadband resonance performance. (b)Sensing characterization of HNA supercell with the thin-film analyte of PMMA and silk protein. The fingerprint absorption peaks ranging from $5.5 \mu \mathrm{m}$ to $9 \mu \mathrm{m}$ are clearly captured by HNA supercell including 
$\mathrm{C}=\mathrm{O}, \mathrm{C}-\mathrm{H}, \mathrm{C}-\mathrm{O}-\mathrm{C}$ bond from PMMA and amide $\mathrm{I}$, amide II and $\mathrm{C}-\mathrm{N}$ bond from silk. (c)Comparison of sensing spectrum of PMMA and silk between HNA and HNA supercell. The HNA from P1, P8, and P16 is selected as a reference with the response to short, medium, and long-wavelength resonance, respectively. It shows HNA supercell has a good response over a broad range of wavelengths from $5.5 \mu \mathrm{m}$ to $9 \mu \mathrm{m}$ which HNA only covers a narrow bandwidth near resonance wavelength for enhancement of fingerprint absorption. (d)I. Schematic drawing of an integrated microfluidic HNA supercell system for liquid sensing. The HNAS on the CaF2 carrier chip is flip bonded to the PDMS surface with the alignment of HNAS into the microfluidic channel. The microfluidic channel is formed by a 3D printed mold and is fixed on a microscope slide. The IR light is shining from the backside of the CaF2 chip, and reflected light is collected to monitor the far-field response of HNA supercell with different aqueous analytes. II. The response of HNAS under the water $(\mathrm{H} 2 \mathrm{O})$ environment. The enhancement of the $\mathrm{O}-\mathrm{H}$ bond of $\mathrm{H} 2 \mathrm{O}$ molecules at $6.0 \mu \mathrm{m}$ is observed at an enhancement factor of. Additionally, the redshift of um from HNAS is observed to demonstrate the refractometry function of HNAS corresponding to the refractive index change of molecules. (e) The dynamic response of HNAS with respect to a different time as acetone injects into water. Each curve indicates the real-time spectrum of a mixed solvent of acetone and water, reflecting in-situ concentration information of acetone and water and dynamic change versus time. (f). Baseline-corrected absorbance spectrum at a different time at board wavelengths range from 2.5 to 3.5 $\mu \mathrm{m}$ for $\mathrm{O}-\mathrm{H}$ bond of water at $2.95 \mu \mathrm{m}$ and 5.5 to $9 \mu \mathrm{m}$ for various fingerprint peaks ( $0-\mathrm{H}$ bond for $\mathrm{H} 2 \mathrm{O}$ at $6.0 \mu \mathrm{m}, \mathrm{C}=\mathrm{O}, \mathrm{C}-\mathrm{H}, \mathrm{C}-\mathrm{C}-\mathrm{C}$ bond for acetone at $5.7 \mu \mathrm{m}, 7.0$ and $7.3 \mu \mathrm{m}, 8.3 \mu \mathrm{m}$, respectively). (g). The integrated absorbance of each fingerprint absorption as a function of time. As time goes by, the absorbance of $\mathrm{O}-\mathrm{H}$ bond of $\mathrm{H} 2 \mathrm{O}$ at $2.95 \mu \mathrm{m}$ and $6.0 \mu \mathrm{m}$ decrease, indicating the concentration decrease of water molecules while the absorbance of $\mathrm{C}=\mathrm{O}, \mathrm{C}-\mathrm{H}, \mathrm{C}-\mathrm{C}-\mathrm{C}$ bond for acetone at $5.7 \mu \mathrm{m}, 7.0$ and $7.3 \mu \mathrm{m}$, $8.3 \mu \mathrm{m}$ increase, representing the concentration increase of acetone molecules. 

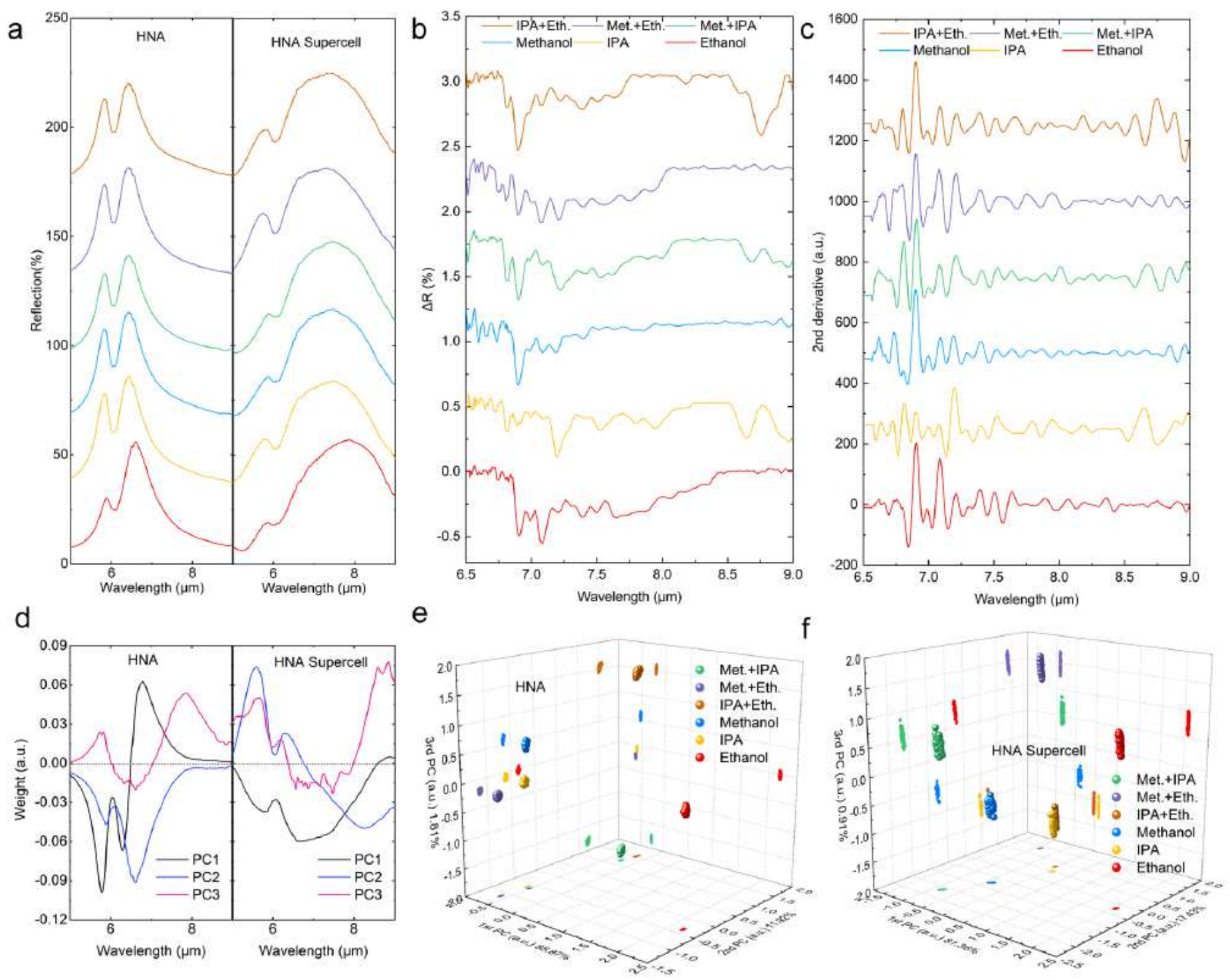

\section{Figure 5}

Machine learning demonstration of HNA spectroscopy by recognition of alcoholic molecules of methanol $(\mathrm{CH} 3 \mathrm{OH})$, ethanol $(\mathrm{C} 2 \mathrm{H} 5 \mathrm{OH})$, and $\mathrm{IPA}(\mathrm{C} 3 \mathrm{H} 7 \mathrm{OH})$ at a concentration of $1 \%$ in water $(\mathrm{H} 2 \mathrm{O})$. (a) The response of HNA spectroscopy under different types of alcoholic molecules. The HNA only response to a narrow bandwidth near resonance wavelength at $\sim 6.5 \mu \mathrm{m}$, while HNA supercell response to a wide bandwidth from $6 \mu \mathrm{m}$ to $9 \mu \mathrm{m}$. The dip at $\sim 6.0 \mu \mathrm{m}$ represents the $\mathrm{O}-\mathrm{H}$ bond of water, which is the common solvent in both cases. (b) The calculated the reflection change spectrum (i) and its second derivative (ii) of HNA supercell under different types of alcoholic molecules from $6.5 \mu \mathrm{m}$ to $9 \mu \mathrm{m}$, showing the fingerprint absorption peaks of each molecule and in a mixture of two types of molecules. (c) The machine learning processed a spectrum of HNA spectroscopy after dimension reduction by principal component analysis. For HNA, the 1st principle component represents the modulation effect of water absorption peaks at 6.0 $\mu \mathrm{m}$. The 2 nd principle component represents the wavelength shift of HNA due to the refractive index of the analyte. The 3rd principle component represents the fingerprint absorption of molecules. While for HNA supercell, the 1 st and 2nd component is flipped as the HNA case indicating the different response 
for refractometry and spectroscopy function. (d) The weight of scores of each spectrum in threedimensional space after PCA for HNA(i) and HNA supercell(ii). Each cluster indicates one type of molecules and their mixtures.
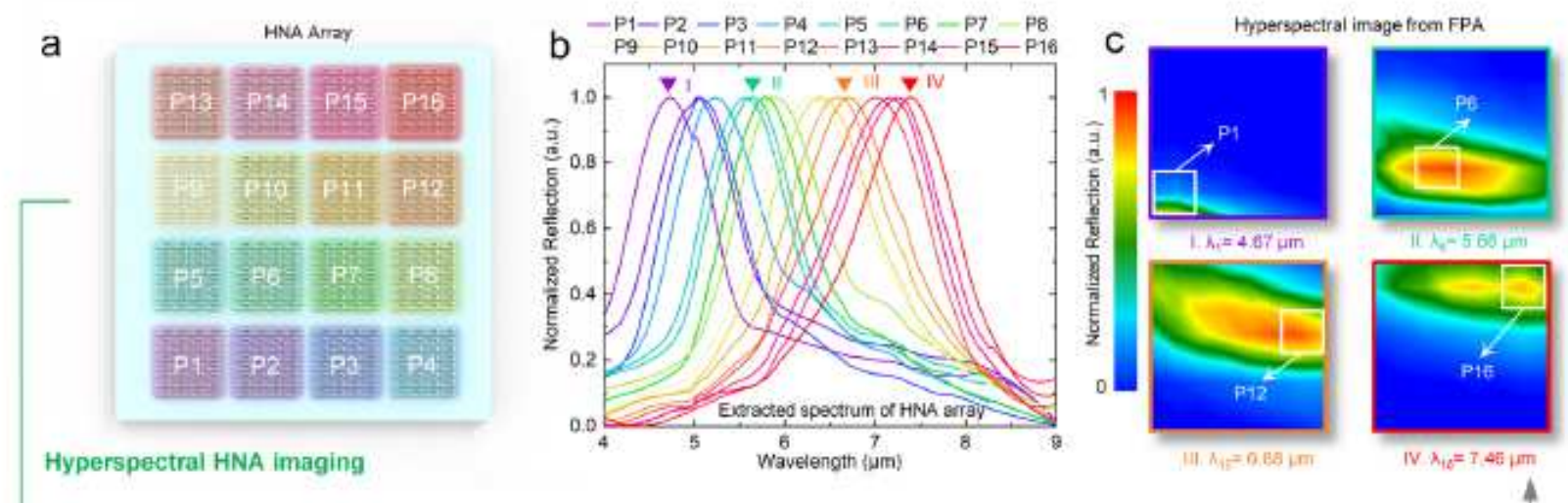

Hyperspectral HNA imaging
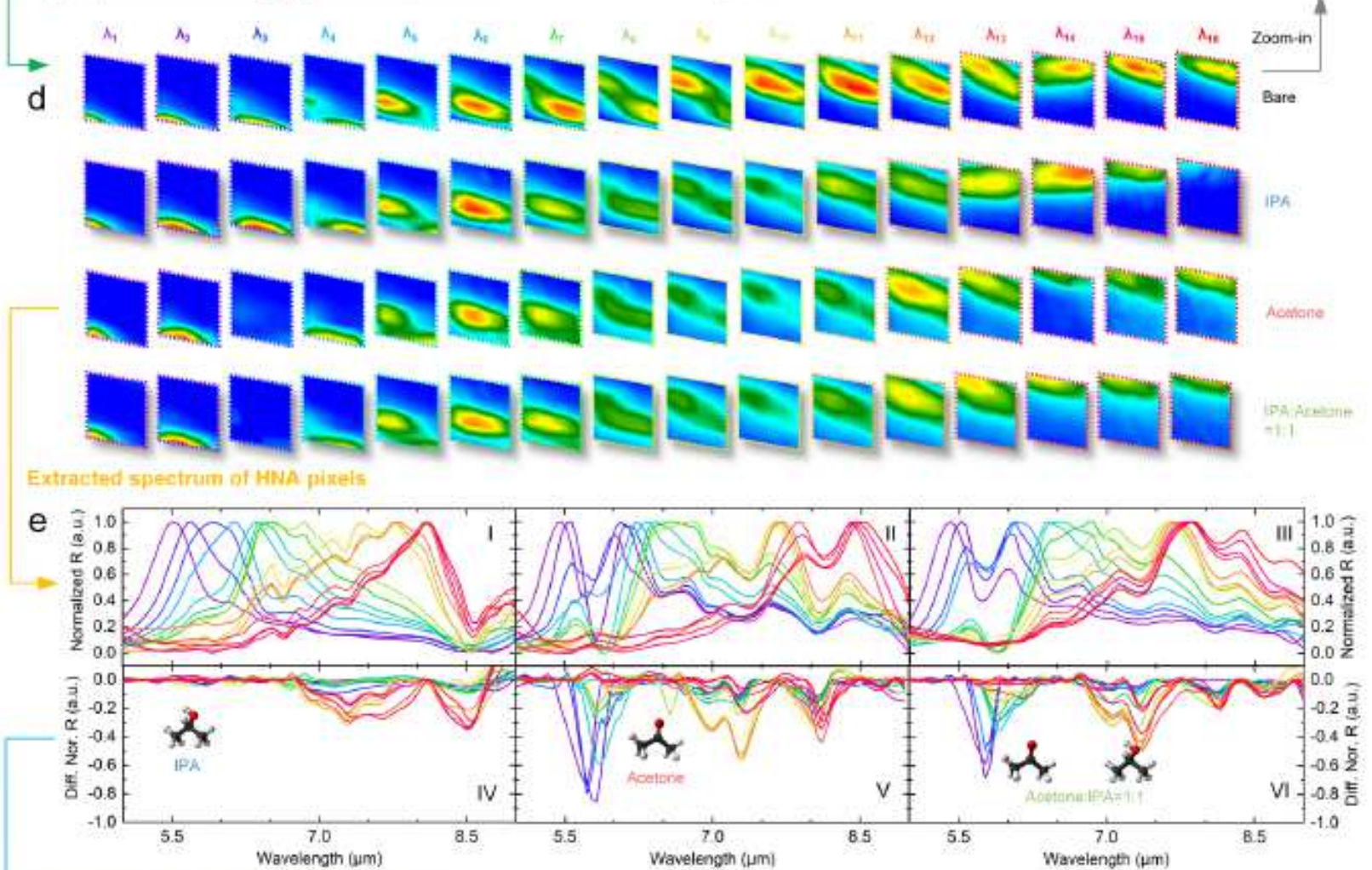

Reconstructed fingerprint barcode imaging

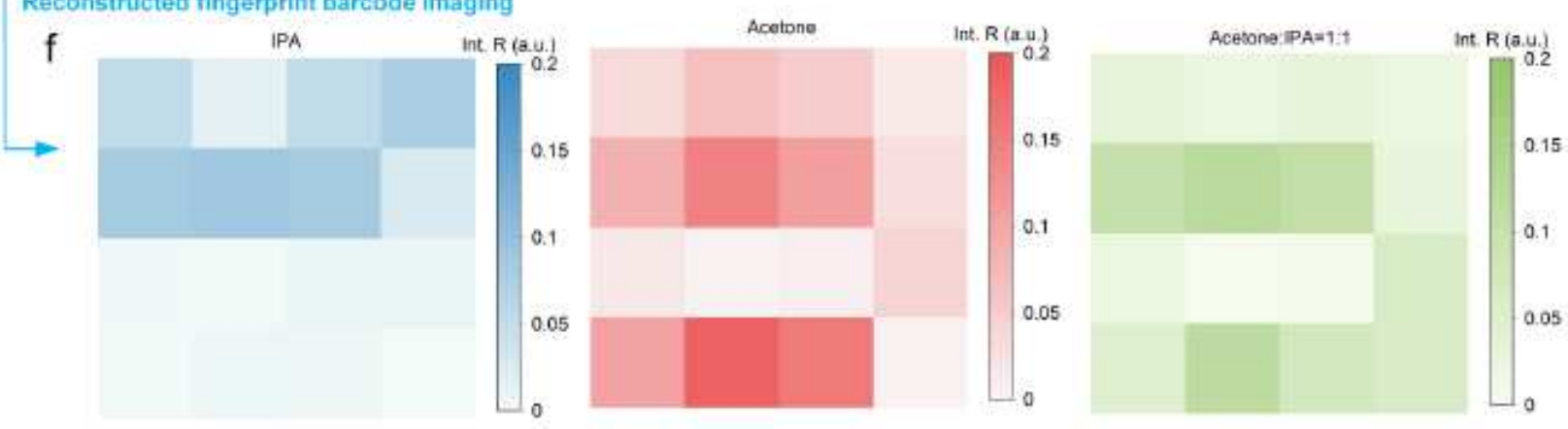

Figure 6

IR fingerprint retrieval and molecules identification by hyperspectral IR imaging for HNA array. (a) Schematic illustration of wavelength gradient HNA array for hyperspectral imaging. Each pixel response 
to different IR wavelengths. (b) By pixelating the wavelength gradient HNA into a four by four arrangement, the hyperspectral imaging is captured by the FPA, representing the different spectrum response of each HNA pixels. P1 response shortest wavelengths $(\sim 4.67 \mu \mathrm{m})$ and P16 response the longest wavelengths $(\sim 7.46 \mu \mathrm{m})$. The wavelength difference is designed to be $\sim 200 \mathrm{~nm}$ to construct a linear gradient in the wavelength domain. (c) Zoom-in picture for the HNA array at four selected wavelengths (i. $4.67 \mu \mathrm{m}$, ii. $5.66 \mu \mathrm{m}$, iii. $6.88 \mu \mathrm{m}$, iv. $7.46 \mu \mathrm{m}$ ). The expected pixel is illuminated at a resonant wavelength while other pixels are dark. The pixel is illuminated at HNA resonance, which are $4.67 \mu \mathrm{m}$ for $\mathrm{P} 1,5.66 \mu \mathrm{m}$ for $\mathrm{P} 6,6.88 \mu \mathrm{m}$ for $\mathrm{P} 12$, and $7.46 \mu \mathrm{m}$ for P16. (d) The hyperspectral image of HNA array at 16 resonance wavelengths for 16 pixels under different liquid analytes conditions (Acetone, IPA and Acetone: IPA=1:1). (e) The extracted normalized reflection and difference normalized reflection spectrum of P1 to P16 under molecules of acetone (I, IV), IPA (II, V), and Acetone: IPA=1:1 (III, VI). The analytes are in the liquid phase and signals are captured by the microfluidic integrated HNA array. At the absorption wavelength of IPA ( $\lambda 7-13$ and $\lambda 16)$ and acetone $(\lambda 3, \lambda 7-11$ and $\lambda 14,15)$, the HNA reflection drops at the desired pixel. (f) The reconstructed fingerprint barcode image by integrating spectrum at working wavelength of each pixel for acetone (I), IPA (II), and Acetone: IPA=1:1 (III). The darker of the pixels refers to the stronger absorption of molecules. The absorption peaks of IPA at 7.0 to $8.0 \mu \mathrm{m}$ and $8.5 \mu \mathrm{m}$ are captured by $\mathrm{P} 9$ to $\mathrm{P} 13$ and $\mathrm{P} 16$ respectively, while the absorption peaks of acetone at $5.74 \mu \mathrm{m}$, 7.0 to $7.5 \mu \mathrm{m}$ and $8.0 \mu \mathrm{m}$ are captured by P1 to P3, P9 to P11 and P14 respectively. In the mixture sample, both absorption peaks are captures from the reconstructed imaging. 